Supporting Information

\title{
Potassium-Catalyzed Hydrosilylation of Activated Olefins: Evidence for Silyl Migration Mechanism
}

\author{
Valeri Leich, Thomas P. Spaniol, and Jun Okuda*,† \\ ${ }^{\dagger}$ Institute of Inorganic Chemistry, RWTH Aachen University, Landoltweg 1, \\ 52056 Aachen, Germany \\ Corresponding Author \\ E-mail jun.okuda@ac.rwth-aachen.de
}




\section{Table of Contents:}

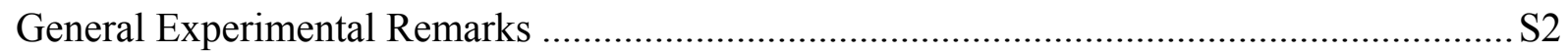

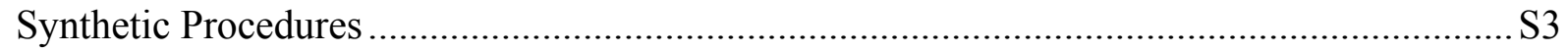

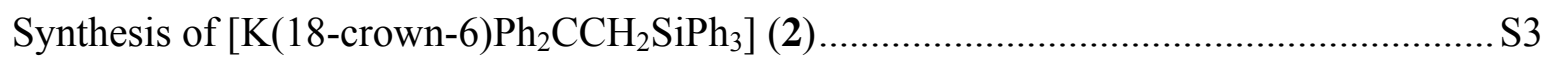

${ }^{1} \mathrm{H},{ }^{13} \mathrm{C}\left\{{ }^{1} \mathrm{H}\right\}$ and ${ }^{29} \mathrm{Si}\left\{{ }^{1} \mathrm{H}\right\}$ NMR spectra of $\left[\mathrm{K}\left(18\right.\right.$-crown-6) $\left.\mathrm{Ph}_{2} \mathrm{CCH}_{2} \mathrm{SiPh}_{3}\right](2) \ldots \ldots \ldots \ldots . . . . \mathrm{S} 4$

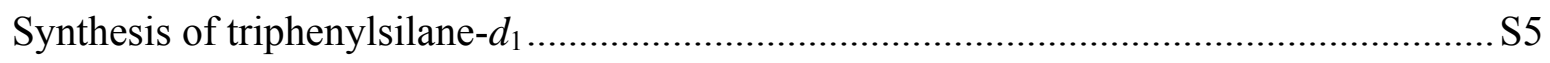

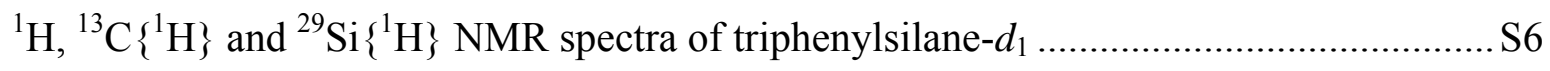

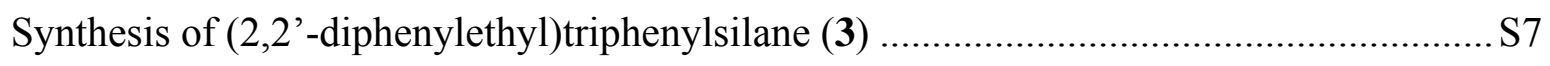

${ }^{1} \mathrm{H},{ }^{13} \mathrm{C}\left\{{ }^{1} \mathrm{H}\right\}$ and ${ }^{29} \mathrm{Si}\left\{{ }^{1} \mathrm{H}\right\}$ NMR spectra of (2,2'-diphenylethyl)triphenylsilane (3) .......... S8

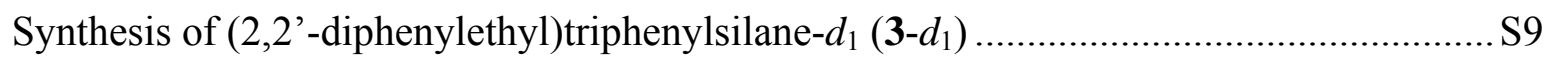

${ }^{1} \mathrm{H},{ }^{13} \mathrm{C}\left\{{ }^{1} \mathrm{H}\right\}$ and ${ }^{29} \mathrm{Si}\left\{{ }^{1} \mathrm{H}\right\}$ NMR spectra of (2,2'-diphenylethyl)triphenylsilane- $d_{1}\left(3-d_{1}\right) \mathrm{S} 10$

Hydrosilylation of alkenes S12

Characterization of $\left(1,2,2^{\prime}\right.$-triphenylethyl)diphenylsilane ........................................... S12

${ }^{1} \mathrm{H},{ }^{13} \mathrm{C}\left\{{ }^{1} \mathrm{H}\right\}$ and ${ }^{29} \mathrm{Si}\left\{{ }^{1} \mathrm{H}\right\}$ NMR spectra of (1,2,2'-triphenylethyl)diphenylsilane............ S13

Characterization of $\left(1,2,2^{\prime}\right.$-triphenylethyl)phenylsilane ............................................... S15

${ }^{1} \mathrm{H},{ }^{13} \mathrm{C}\left\{{ }^{1} \mathrm{H}\right\}$ and ${ }^{29} \mathrm{Si}\left\{{ }^{1} \mathrm{H}\right\}$ NMR spectra of (1,2,2'-triphenylethyl)phenylsilane............... S16

in situ ${ }^{1} \mathrm{H}$ NMR Spectra of stoichiometric reactions ..................................................... S17

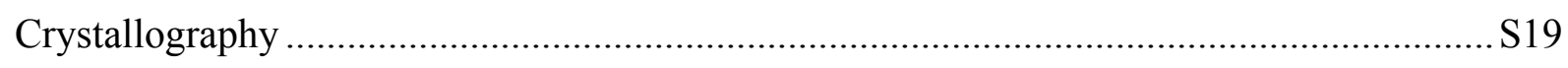

Crystal structure determination of $\left[\mathrm{K}\left(18\right.\right.$-crown-6) $\left.\mathrm{Ph}_{2} \mathrm{CCH}_{2} \mathrm{SiPh}_{3}\right](2)$......................... S19

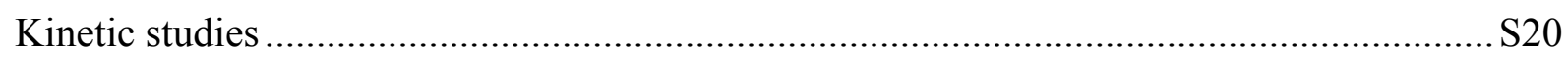

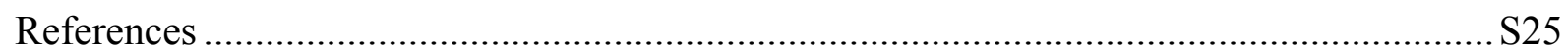




\section{General Experimental Remarks}

All operations were performed under an inert atmosphere of dry argon using standard Schlenk line or glove box techniques. THF- $d_{8}$ and benzene were distilled under argon from sodium/benzophenone ketyl prior to use. THF and pentane were purified using a MB SPS- 800 solvent purification system. 1,4,7,10,13,16-hexaoxacyclooctadecane (18-crown-6), $\mathrm{Ph}_{3} \mathrm{SiCl}$, $\mathrm{LiAlD}_{4}, \quad \mathrm{HSiPh}_{3}, \quad \mathrm{H}_{2} \mathrm{SiPh}_{2}, \quad \mathrm{H}_{3} \mathrm{SiPh}, \quad$ 1,1-diphenylethylene, 1,3-cyclohexadiene, triphenylethylene, 1-phenylcyclohexene, 4-phenyl-1-butene and trans, trans-1,4-diphenyl-1,3butadiene were purchased from Sigma Aldrich and purified by vacuum sublimation or distillation prior to use.

Elemental analyses were performed on an elementar vario EL machine. CI-MS spectra were recorded on a Varian MAT 212S or Finnigan SSQ 7000 machine. Metal content was determined by atom absorption spectroscopy on a Shimadzu AA-6200 ( $\lambda=460.7 \mathrm{~nm}$, air/acetylene flame) machine. ${ }^{1} \mathrm{H},{ }^{13} \mathrm{C}\left\{{ }^{1} \mathrm{H}\right\}$ and ${ }^{29} \mathrm{Si}\left\{{ }^{1} \mathrm{H}\right\}$ NMR spectra were recorded on a Bruker Avance II 400 or a Bruker Avance III HD 400 spectrometer at $25{ }^{\circ} \mathrm{C}$ in J. Young type NMR tubes. ${ }^{2} \mathrm{H}$ NMR spectra were recorded on an Agilent VNMRS 400 spectrometer. Chemical shifts for ${ }^{1} \mathrm{H},{ }^{13} \mathrm{C}\left\{{ }^{1} \mathrm{H}\right\}$, and ${ }^{29} \mathrm{Si}\left\{{ }^{1} \mathrm{H}\right\}$ NMR spectra were referenced internally using the residual solvent resonance and are reported relative to tetramethylsilane. The resonances in ${ }^{1} \mathrm{H}$ and ${ }^{13} \mathrm{C}$ NMR spectra were assigned on the basis of two-dimensional NMR experiments (COSY, HSQC, HMBC).

$\left[\mathrm{K}\left(18 \text {-crown-6) } \mathrm{SiPh}_{3}\right]^{\mathrm{S} 1}\right.$ was prepared according to a published procedure. Triphenylsilane- $d_{1}{ }^{\mathrm{S} 2}$ and (2,2'-diphenylethyl)triphenylsilane ${ }^{\mathrm{S} 3}$ are literature known compounds, however missing data was completed. 


\section{Synthetic Procedures}

\section{Synthesis of [K(18-crown-6) $\left.\mathrm{Ph}_{2} \mathrm{CCH}_{2} \mathrm{SiPh}_{3}\right]$ (2)}

A solution of $\left[\mathrm{K}\left(18\right.\right.$-crown-6) $\left.\mathrm{SiPh}_{3}\right](\mathbf{1})(480 \mathrm{mg}, 0.85 \mathrm{mmol})$ in THF $(4 \mathrm{~mL})$ was added to 1,1'-DPE (153 mg, $0.85 \mathrm{mmol})$ in THF $(4 \mathrm{~mL})$ and stirred for $5 \mathrm{~min}$ at $25^{\circ} \mathrm{C}$. The reaction mixture turned red immediately, was layered with pentane, and red crystals grew within $12 \mathrm{~h}$ at $-30{ }^{\circ} \mathrm{C}$. The supernatant was decanted off and the red powder washed with pentane $(3 \times 3$ $\mathrm{mL})$. After drying in vacuo, [K(18-crown-6) $\left.\mathrm{Ph}_{2} \mathrm{CCH}_{2} \mathrm{SiPh}_{3}\right]$ (2) (584 mg, 0.79 mmol, 93 \%) was isolated as red powder. Single crystals of 2 suitable for X-ray diffraction were grown from a concentrated benzene solution at $25^{\circ} \mathrm{C}$ over a period of $24 \mathrm{~h}$.

${ }^{1} \mathrm{H}$ NMR (THF-d $\left.d_{8}, 400.1 \mathrm{MHz}\right): \delta 2.70$ (s, 2H, $\mathrm{CH}_{2}$ ), 3.47 (s, 24H, 18-crown-6), 5.49 (tt, 2H, ${ }^{3} J_{\mathrm{H}, \mathrm{H}}=6.85 \mathrm{~Hz},{ }^{4} J_{\mathrm{H}, \mathrm{H}}=0.98 \mathrm{~Hz}$, para- $\left.\mathrm{Ph}_{\mathrm{DPE}}\right), 6.32\left(\mathrm{dd}, 4 \mathrm{H},{ }^{3} J_{\mathrm{H}, \mathrm{H}}=8.56 \mathrm{~Hz},{ }^{3} J_{\mathrm{H}, \mathrm{H}}=6.85 \mathrm{~Hz}\right.$, meta-Ph $\left.\mathrm{DPE}_{\mathrm{DPE}}\right), 6.82\left(\mathrm{dd}, 4 \mathrm{H},{ }^{3} J_{\mathrm{H}, \mathrm{H}}=8.56 \mathrm{~Hz},{ }^{4} J_{\mathrm{H}, \mathrm{H}}=0.98 \mathrm{~Hz}\right.$, ortho-Ph $\left.\mathrm{DPE}_{\mathrm{DE}}\right), 7.11-7.21(\mathrm{~m}, 9 \mathrm{H}$, para/meta- $\left.\mathrm{Ph}_{\mathrm{Si}}\right)$, 7.53-7.57 (m, 6H, ortho- $\left.\mathrm{Ph}_{\mathrm{Si}}\right) \mathrm{ppm}$.

${ }^{13} \mathrm{C}\left\{{ }^{1} \mathrm{H}\right\}$ NMR (THF-d, $\left.100.6 \mathrm{MHz}\right): \delta 21.16\left(\mathrm{Ph}_{2} \mathrm{CCH}_{2} \mathrm{SiPh}_{3}\right), 71.08$ (18-K-6), 76.49 $\left(\mathrm{Ph}_{2} \mathrm{CCH}_{2} \mathrm{SiPh}_{3}\right.$ ), 107.03 (para-Ph ${ }_{\mathrm{DPE}}$ ), 118.37 (ortho-Ph $\mathrm{DPE}_{\mathrm{DP}}$ ), 127.63 (meta- $\mathrm{Ph}_{\mathrm{Si}}$ ), 127.89

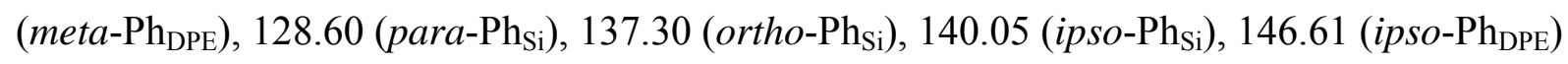
ppm.

${ }^{29} \mathrm{Si}\left\{{ }^{1} \mathrm{H}\right\}$ NMR $\left(\mathrm{THF}-d_{8}, 79.5 \mathrm{MHz}\right): \delta-17.35\left(\mathrm{Ph}_{2} \mathrm{CCH}_{2} \mathrm{SiPh}_{3}\right) \mathrm{ppm}$.

Anal. Calcd for $\mathrm{C}_{44} \mathrm{H}_{51} \mathrm{KO}_{6} \mathrm{Si}\left(743.06 \mathrm{~g} \cdot \mathrm{mol}^{-1}\right)$ : C, 71.12; H, 6.92; K, 5.26. Found: C, 68.31; $\mathrm{H}, 6.88 ; \mathrm{K}, 5.25 \%$. 
${ }^{1} \mathrm{H},{ }^{13} \mathrm{C}\left\{{ }^{1} \mathrm{H}\right\}$ and ${ }^{29} \mathrm{Si}\left\{{ }^{1} \mathrm{H}\right\}$ NMR spectra of [K(18-crown-6) $\left.\mathrm{Ph}_{2} \mathrm{CCH}_{2} \mathrm{SiPh}_{3}\right]$ (2)

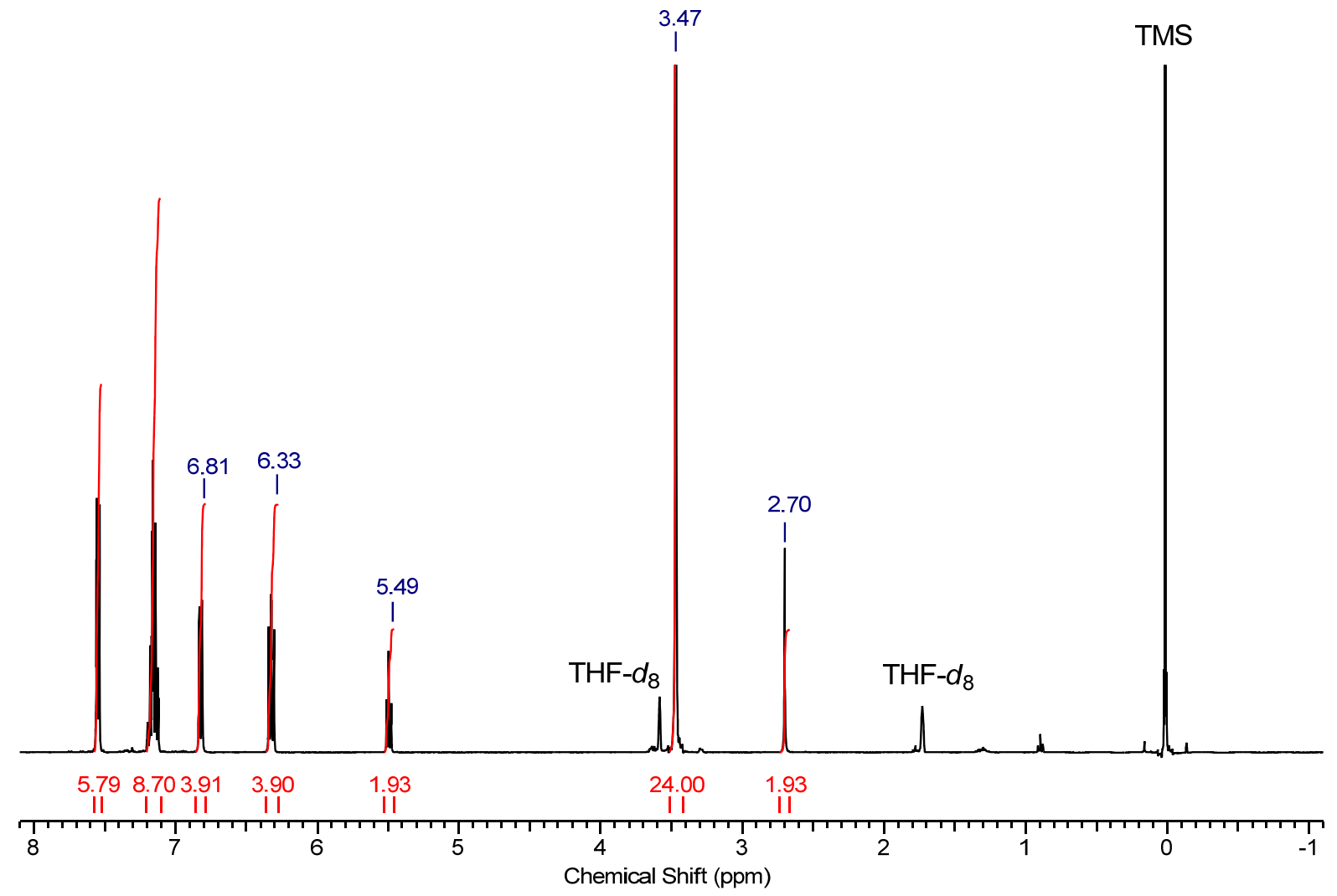

Figure S1. ${ }^{1} \mathrm{H}$ NMR spectrum of $\left[\mathrm{K}\left(18\right.\right.$-crown-6) $\left.\mathrm{Ph}_{2} \mathrm{CCH}_{2} \mathrm{SiPh}_{3}\right]$ in THF- $d_{8}$ at $25^{\circ} \mathrm{C}$.

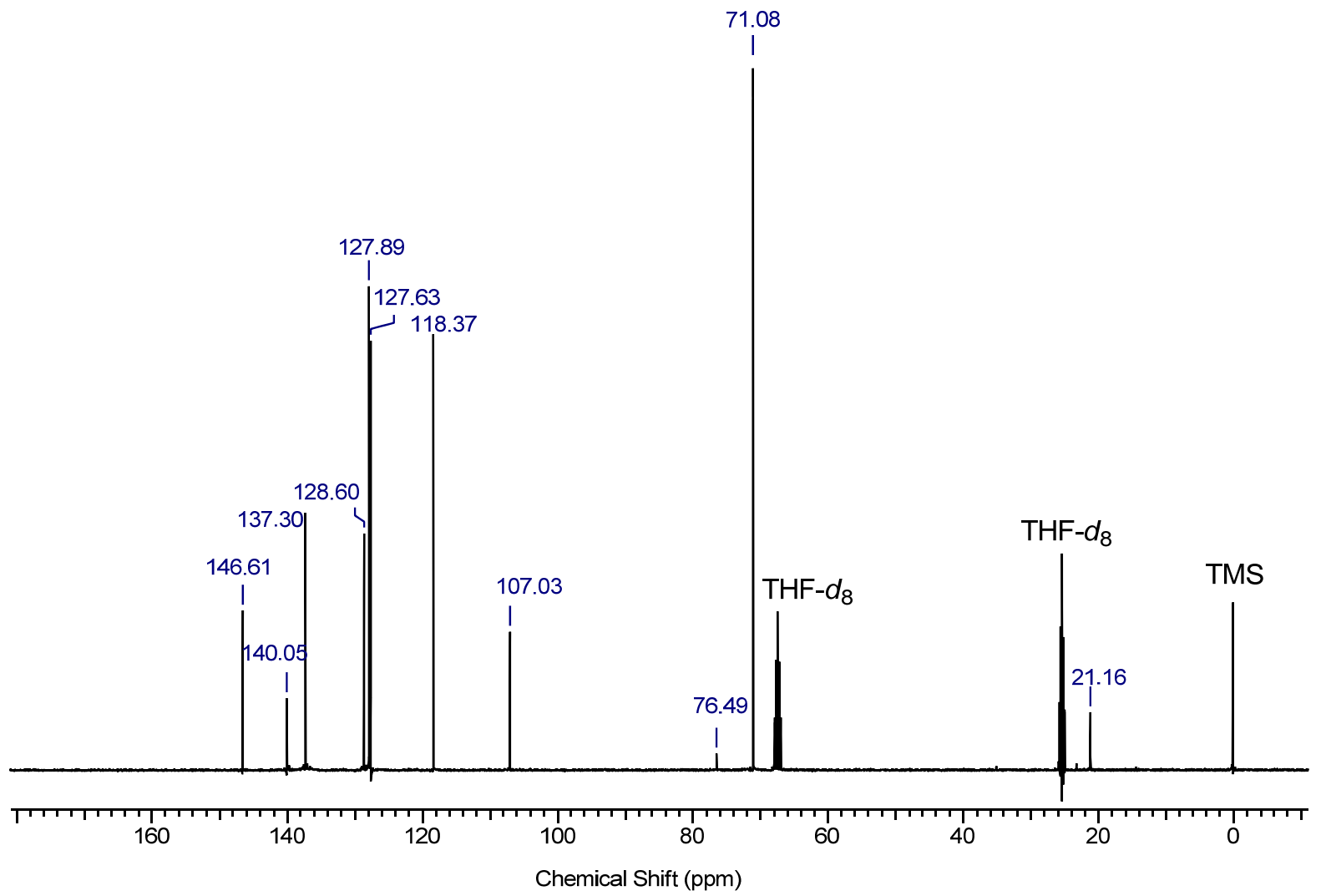

Figure S2. ${ }^{13} \mathrm{C}\left\{{ }^{1} \mathrm{H}\right\}$ NMR spectrum of $\left[\mathrm{K}\left(18\right.\right.$-crown-6) $\left.\mathrm{Ph}_{2} \mathrm{CCH}_{2} \mathrm{SiPh}_{3}\right]$ in THF- $d_{8}$ at $25{ }^{\circ} \mathrm{C}$. 


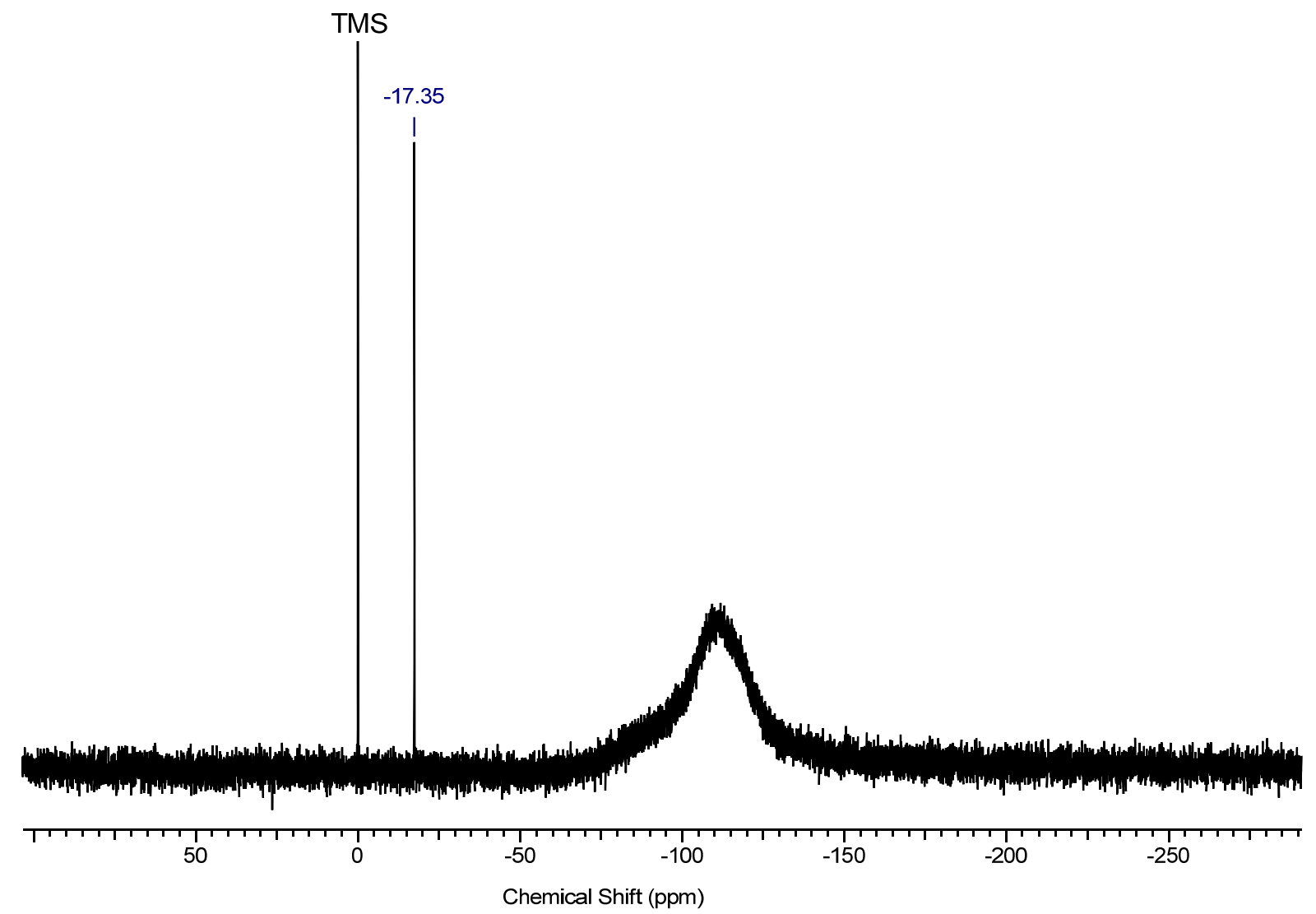

Figure $\mathrm{S} 3 .{ }^{29} \mathrm{Si}\left\{{ }^{1} \mathrm{H}\right\}$ NMR spectrum of $\left[\mathrm{K}\left(18\right.\right.$-crown-6) $\left.\mathrm{Ph}_{2} \mathrm{CCH}_{2} \mathrm{SiPh}_{3}\right]$ in $\mathrm{THF}-d_{8}$ at $25{ }^{\circ} \mathrm{C}$.

\section{Synthesis of triphenylsilane- $d_{1}{ }^{\text {S2 }}$}

A solution of $\mathrm{Ph}_{3} \mathrm{SiCl}(2.95 \mathrm{~g}, 10 \mathrm{mmol})$ in THF $(10 \mathrm{~mL})$ was added to a suspension of $\mathrm{LiAlD}_{4}(0.42 \mathrm{~g}, 10 \mathrm{mmol})$ in THF $(30 \mathrm{~mL})$ at $-30{ }^{\circ} \mathrm{C}$. Die reaction mixture was stirred at -30 ${ }^{\circ} \mathrm{C}$ for $2 \mathrm{~h}$ and warmed to $25{ }^{\circ} \mathrm{C}$ over a period of $24 \mathrm{~h}$. The solvent was removed under reduced pressure, the product extracted with pentane and the resulting suspension filtered over a celite column. Pentane was removed under reduced pressure and $\mathrm{DSiPh}_{3}(1.64 \mathrm{~g}, 6.3 \mathrm{mmol}$, $63 \%)$ was isolated as colorless powder after vacuum sublimation $\left(T=100{ }^{\circ} \mathrm{C}, p=1 \times 10^{-3}\right.$ mbar).

${ }^{1} \mathrm{H}$ NMR $\left(\right.$ THF- $\left.d_{8}, 400.1 \mathrm{MHz}\right): \delta$ 7.27-7.52 (m, 9H, para/meta- $\left.\mathrm{Ph}_{\mathrm{Si}}\right), 7.56-7.67(\mathrm{~m}, 6 \mathrm{H}$, ortho- $\left.\mathrm{Ph}_{\mathrm{Si}}\right) \mathrm{ppm}$.

${ }^{13} \mathrm{C}\left\{{ }^{1} \mathrm{H}\right\}$ NMR $\left(\mathrm{THF}-d_{8}, 100.6 \mathrm{MHz}\right): \delta 128.91\left(\right.$ meta- $\left.\mathrm{Ph}_{\mathrm{Si}}\right), 130.67$ (para- $\left.\mathrm{Ph}_{\mathrm{Si}}\right), 134.30$ (ipso$\mathrm{Ph}_{\mathrm{Si}}$ ), 136.61 (ortho- $\mathrm{Ph}_{\mathrm{Si}}$ ) ppm.

${ }^{29} \mathrm{Si}\left\{{ }^{1} \mathrm{H}\right\}$ NMR (THF- $\left.d_{8}, 79.5 \mathrm{MHz}\right): \delta-18.19\left(\mathrm{t},{ }^{1} J_{\mathrm{D}, \mathrm{Si}}=30.32 \mathrm{~Hz}, \mathrm{Ph}_{3} \mathrm{SiD}\right) \mathrm{ppm}$.

${ }^{2} \mathrm{H}$ NMR (THF-d, $\left.61.1 \mathrm{MHz}\right): \delta 5.63\left(\mathrm{~d}, 1 \mathrm{D},{ }^{1} J_{\mathrm{D}, \mathrm{Si}}=16.15 \mathrm{~Hz}, \mathrm{Ph}_{3} \mathrm{Si} D\right) \mathrm{ppm}$.

CI MS (100 eV) m/z: $261\left(\mathrm{M}^{+}, 100 \%\right), 182$ (83), 77 (7). 
${ }^{1} \mathrm{H},{ }^{13} \mathrm{C}\left\{{ }^{1} \mathrm{H}\right\}$ and ${ }^{29} \mathrm{Si}\left\{{ }^{1} \mathrm{H}\right\}$ NMR spectra of triphenylsilane- $d_{1}$

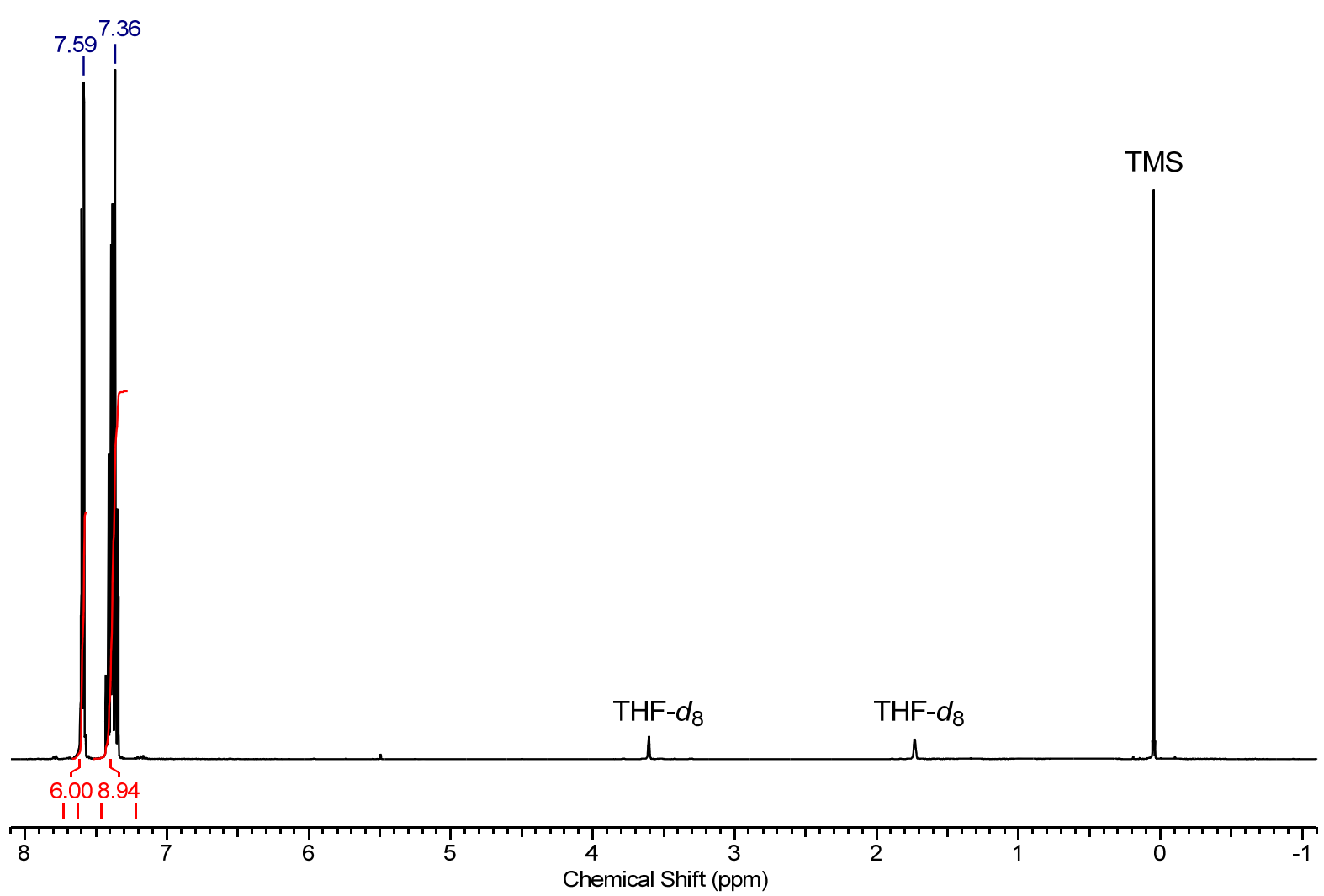

Figure S4. ${ }^{1} \mathrm{H}$ NMR spectrum of triphenylsilane- $d_{1}$ in THF- $d_{8}$ at $25{ }^{\circ} \mathrm{C}$.

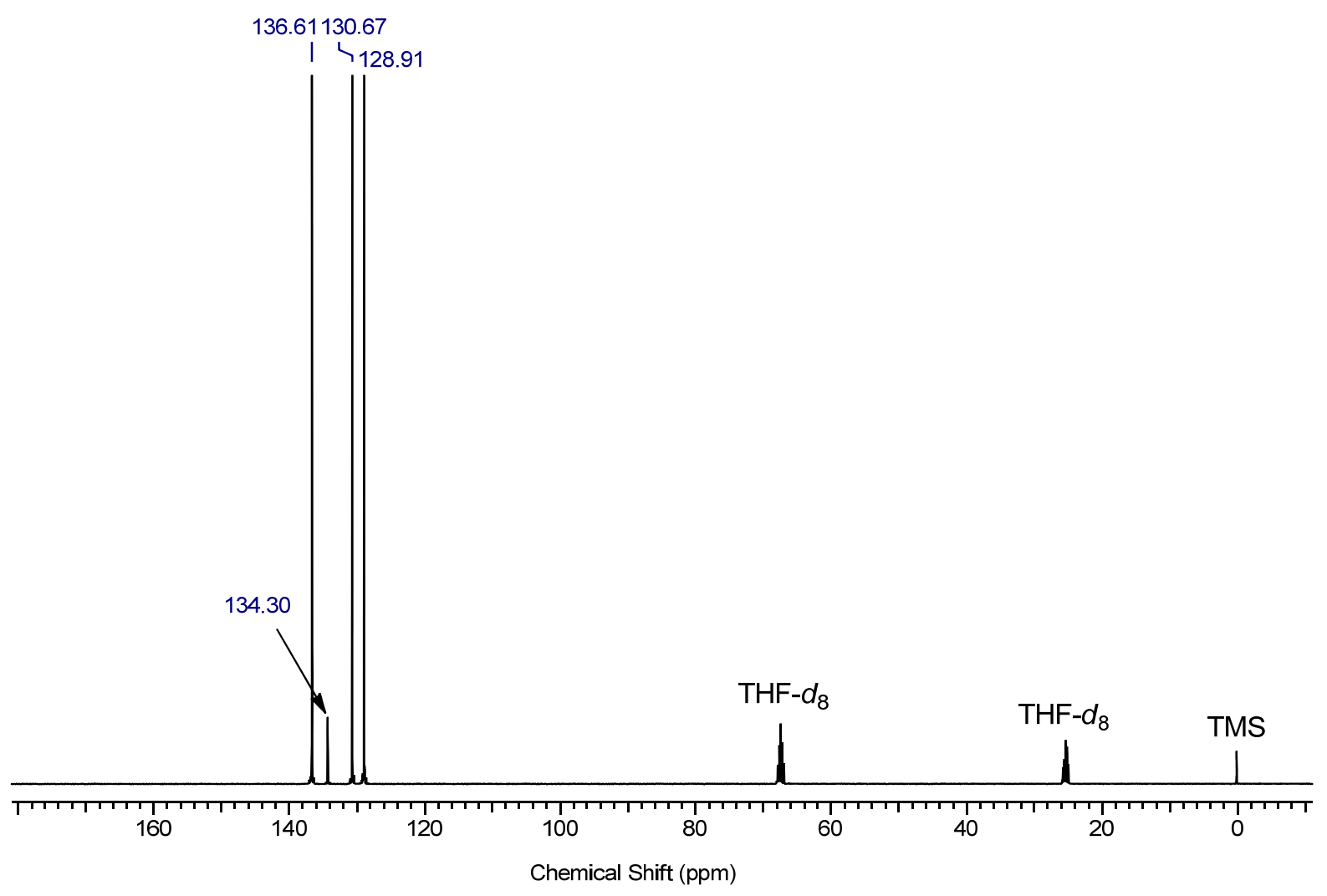

Figure S5. ${ }^{13} \mathrm{C}\left\{{ }^{1} \mathrm{H}\right\}$ NMR spectrum of triphenylsilane- $d_{1}$ in THF- $d_{8}$ at $25{ }^{\circ} \mathrm{C}$. 


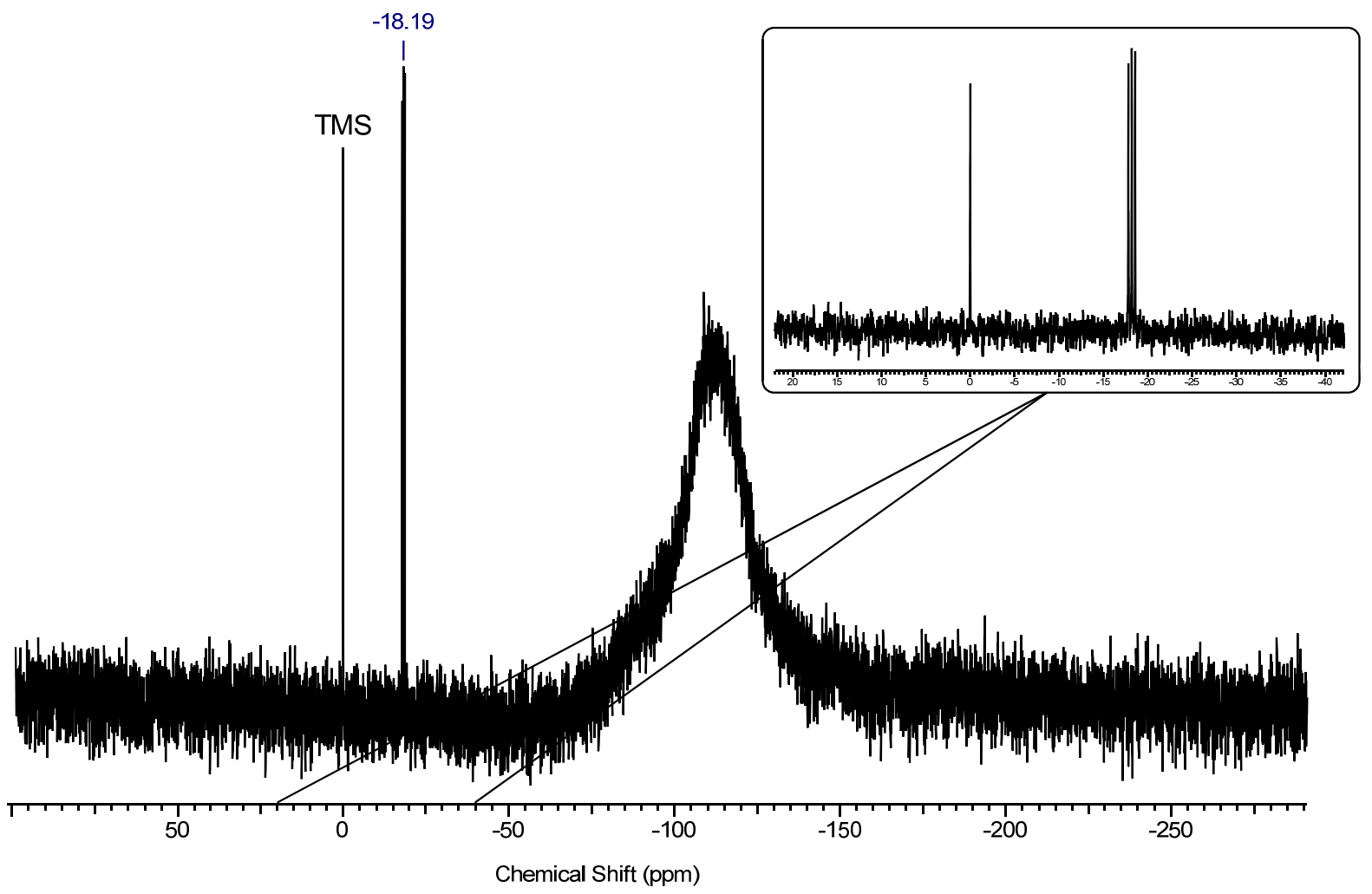

Figure S6. ${ }^{29} \mathrm{Si}\left\{{ }^{1} \mathrm{H}\right\}$ NMR spectrum of triphenylsilane- $d_{1}$ in THF- $d_{8}$ at $25{ }^{\circ} \mathrm{C}$.

\section{Synthesis of (2,2'-diphenylethyl)triphenylsilane (3) ${ }^{\mathrm{S3}}$}

A solution of 1,1'-DPE (180 mg. $1.00 \mathrm{mmol})$ and [K(18-crown-6)SiPh 3 (14 mg, $25 \mu \mathrm{mol})$ in THF (2 mL) was added to a solution of $\mathrm{Ph}_{3} \mathrm{SiH}(286 \mathrm{mg}, 1.10 \mathrm{mmol})$ in THF $(6 \mathrm{~mL})$ and stirred at $60{ }^{\circ} \mathrm{C}$ over a period of $24 \mathrm{~h}$. The reaction mixture was quenched with water and extracted with $\mathrm{Et}_{2} \mathrm{O}(3 \times 20 \mathrm{~mL})$. The organic phases were dried over $\mathrm{Na}_{2} \mathrm{SO}_{4}$ and the solvent was removed under reduced pressure. After crystallization from $\mathrm{EtOH} / \mathrm{Et}_{2} \mathrm{O}$ and drying in vacuo, (2,2'-diphenylethyl)triphenylsilane (3) (350 mg, $0.79 \mathrm{mmol}, 79 \%$ ) was isolated as colorless crystals. Mp $107.2{ }^{\circ} \mathrm{C}$ (lit.: $\left.{ }^{\mathrm{S} 3} 106-108^{\circ} \mathrm{C}\right)$.

${ }^{1} \mathrm{H}$ NMR (THF-d, $400.1 \mathrm{MHz}$ ): $\delta 2.33$ (d, 2H, $\mathrm{Ph}_{2} \mathrm{CHCH}_{2} \mathrm{SiPh}_{3},{ }^{3} \mathrm{~J}_{\mathrm{H}, \mathrm{H}}=7.28 \mathrm{~Hz}$ ), $4.24(\mathrm{t}, 1 \mathrm{H}$, $\mathrm{Ph}_{2} \mathrm{CHCH}_{2} \mathrm{SiPh}_{3},{ }^{3} J_{\mathrm{H}, \mathrm{H}}=7.28 \mathrm{~Hz}$ ), 6.97-7.03 (m, 2H, para-Ph ${ }_{\mathrm{DPE}}$ ), 7.04-7.10 (m, 4H, meta$\mathrm{Ph}_{\mathrm{DPE}}$ ), 7.12-7.17 (m, 4H, ortho-Ph $\left.\mathrm{DPE}\right)$, 7.19-7.26 (m, 6H, meta-Ph $\mathrm{Si}_{\mathrm{i}}$ ), 7.27-7.33 (m, 3H, para- $\left.-\mathrm{Ph}_{\mathrm{Si}}\right), 7.33-7.37\left(\mathrm{~m}, 6 \mathrm{H}\right.$, ortho- $\left.-\mathrm{Ph}_{\mathrm{Si}}\right) \mathrm{ppm}$.

${ }^{13} \mathrm{C}\left\{{ }^{1} \mathrm{H}\right\}$ NMR (THF-d $\left.d_{8}, 100.6 \mathrm{MHz}\right): \delta 22.18\left(\mathrm{Ph}_{2} \mathrm{CHCH}_{2} \mathrm{SiPh}_{3}\right), 48.14\left(\mathrm{Ph}_{2} \mathrm{CHCH}_{2} \mathrm{SiPh}_{3}\right)$,

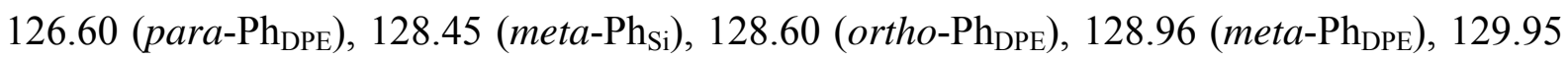

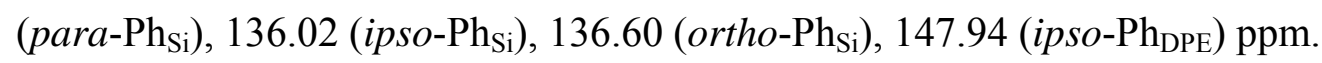

${ }^{29} \mathrm{Si}\left\{{ }^{1} \mathrm{H}\right\}$ NMR (THF-d, $\left.79.5 \mathrm{MHz}\right): \delta-11.25\left(\mathrm{Ph}_{2} \mathrm{CHCH}_{2} \mathrm{SiPh}_{3}\right) \mathrm{ppm}$. 
Anal. Calcd for $\mathrm{C}_{32} \mathrm{H}_{28} \mathrm{Si}\left(440.65 \mathrm{~g} \cdot \mathrm{mol}^{-1}\right)$ : C, 87.22; H, 6.40. Found: C, 86.93; H, 6.35\%.

CI MS (100 eV) m/z: $363\left(\mathrm{M}^{+}-\mathrm{C}_{6} \mathrm{H}_{5}, 100 \%\right), 259$ (30); molecular peak could not be detected.

\section{${ }^{1} \mathrm{H},{ }^{13} \mathrm{C}\left\{{ }^{1} \mathrm{H}\right\}$ and ${ }^{29} \mathrm{Si}\left\{{ }^{1} \mathrm{H}\right\}$ NMR spectra of (2,2'-diphenylethyl)triphenylsilane (3)}

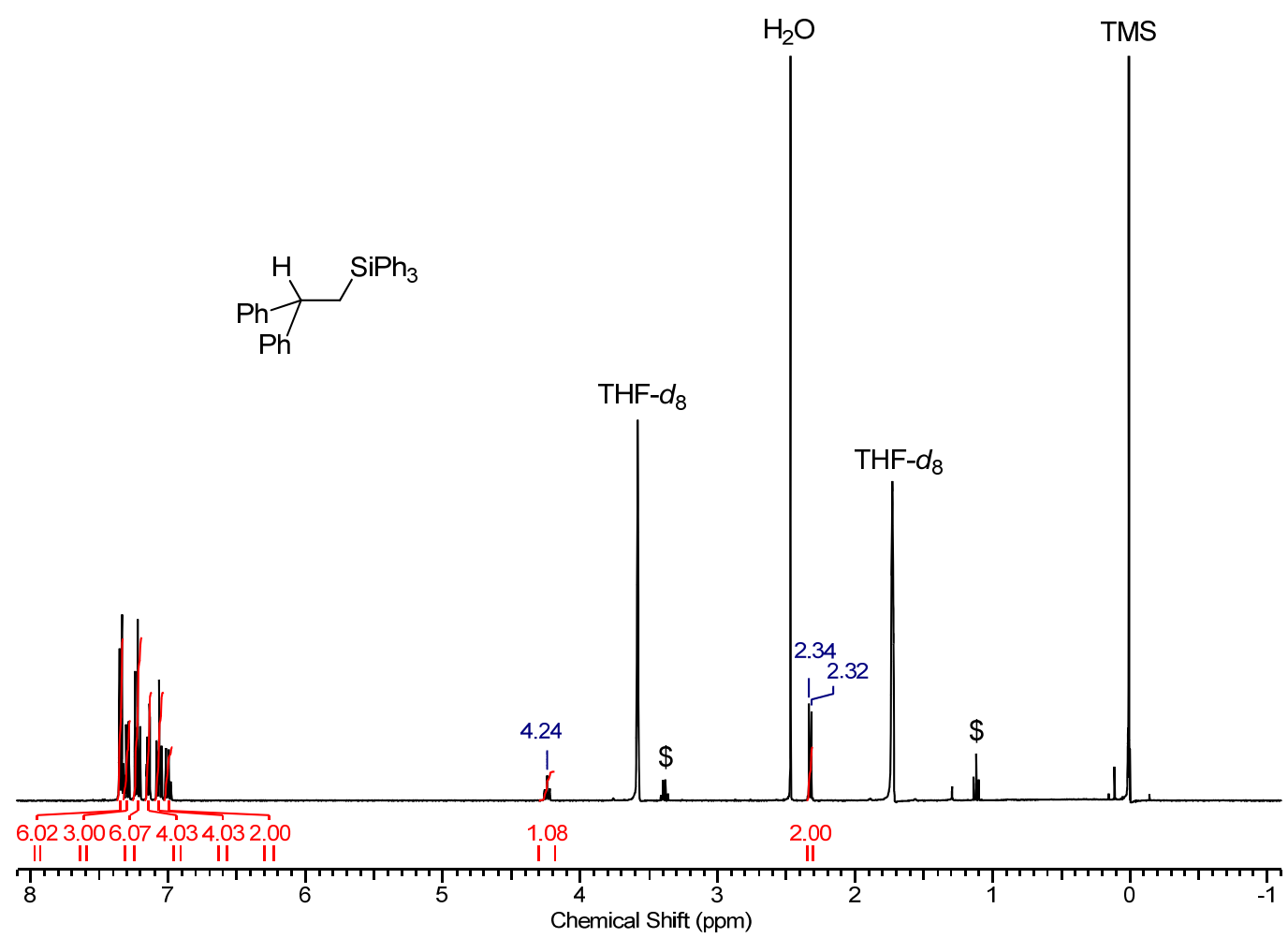

Figure S7. ${ }^{1} \mathrm{H}$ NMR spectrum of (2,2'-diphenylethyl)triphenylsilane in THF- $d_{8}$ at $25{ }^{\circ} \mathrm{C}$. \$ denotes traces of $\mathrm{Et}_{2} \mathrm{O}$. Residual $\mathrm{H}_{2} \mathrm{O}$ from THF- $d_{8}$.

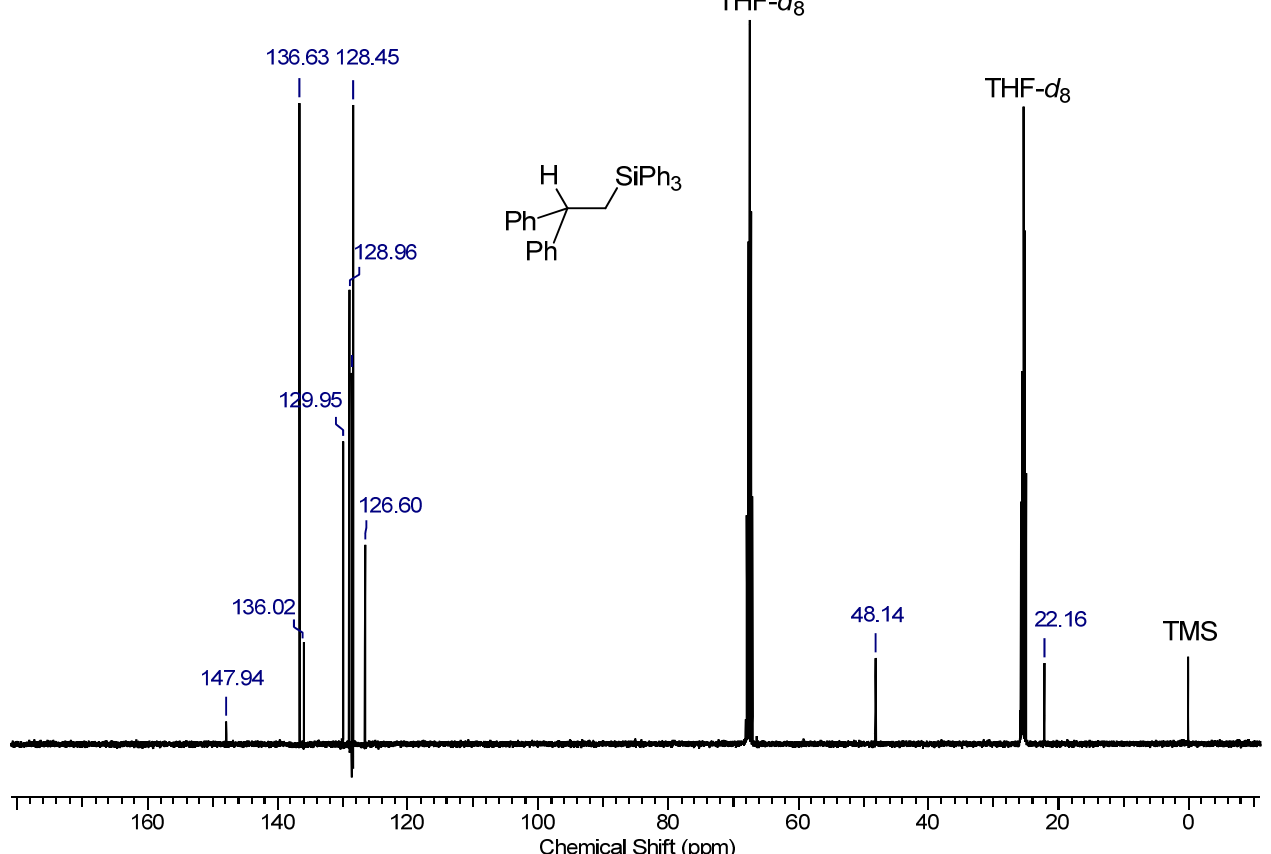

Figure S8. ${ }^{13} \mathrm{C}\left\{{ }^{1} \mathrm{H}\right\}$ NMR spectrum of (2,2'-diphenylethyl)triphenylsilane in THF- $d_{8}$ at $25{ }^{\circ} \mathrm{C}$. 


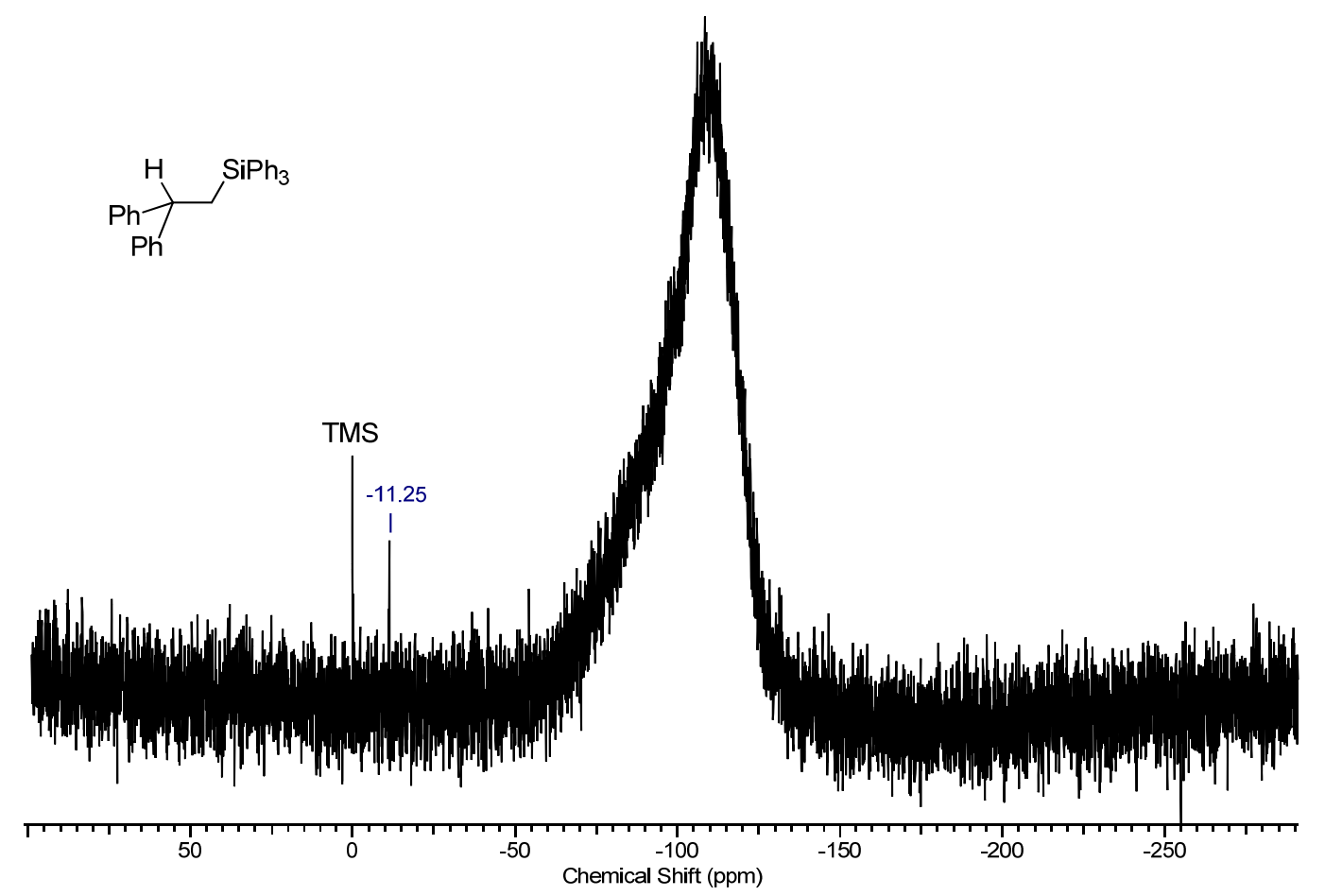

Figure S9. ${ }^{29} \mathrm{Si}\left\{{ }^{1} \mathrm{H}\right\}$ NMR spectrum of (2,2'-diphenylethyl)triphenylsilane in THF- $d_{8}$ at 25 ${ }^{\circ} \mathrm{C}$.

\section{Synthesis of (2,2'-diphenylethyl)triphenylsilane- $d_{1}\left(3-d_{1}\right)$}

A solution of 1,1'-DPE (180 mg. $1.00 \mathrm{mmol})$ and [K(18-crown-6) $\left.\mathrm{SiPh}_{3}\right]$ (14 mg, $\left.25 \mu \mathrm{mol}\right)$ in THF $(2 \mathrm{~mL})$ was added to a solution of $\mathrm{Ph}_{3} \mathrm{SiD}(287 \mathrm{mg}, 1.10 \mathrm{mmol})$ in THF $(6 \mathrm{~mL})$ and stirred at $60{ }^{\circ} \mathrm{C}$ over a period of $5 \mathrm{~d}$. The reaction mixture was quenched with water and extracted with $\mathrm{Et}_{2} \mathrm{O}(3 \times 20 \mathrm{~mL})$. The organic phases were dried over $\mathrm{Na}_{2} \mathrm{SO}_{4}$ and the solvent was removed under reduced pressure. After crystallization from $\mathrm{EtOH} / \mathrm{Et}_{2} \mathrm{O}$ and drying in vacuo, (2,2'-diphenylethyl)triphenylsilane- $d_{1}\left(3-d_{1}\right)(324 \mathrm{mg}, 0.73 \mathrm{mmol}, 73 \%)$ was isolated as colorless crystals.

${ }^{1} \mathrm{H}$ NMR (THF-d $\left.d_{8}, 400.1 \mathrm{MHz}\right): \delta 2.32\left(\mathrm{~s}, 2 \mathrm{H}, \mathrm{Ph}_{2} \mathrm{CDCH}_{2} \mathrm{SiPh}_{3}\right.$ ), 6.97-7.03 (m, 2H, para$\mathrm{Ph}_{\mathrm{DPE}}$ ), 7.03-7.10 (m, 4H, meta-Ph $\left.\mathrm{DPE}\right)$, 7.12-7.17 (m, 4H, ortho-Ph $\mathrm{DPE}_{\text {) }}$, 7.19-7.26 (m, 6H, meta-Ph $\mathrm{Si}_{\mathrm{Si}}$ ), 7.27-7.33 (m, 3H, para-Ph $\left.\mathrm{Si}\right), 7.33-7.38\left(\mathrm{~m}, 6 \mathrm{H}\right.$, ortho- $\left.\mathrm{Ph}_{\mathrm{Si}}\right) \mathrm{ppm}$.

${ }^{13} \mathrm{C}\left\{{ }^{1} \mathrm{H}\right.$ NMR (THF- $\left.d_{8}, 100.6 \mathrm{MHz}\right): \delta 22.18\left(\mathrm{Ph}_{2} \mathrm{CDCH}_{2} \mathrm{SiPh}_{3}\right), 126.73$ (para-Ph $\left.\mathrm{PPE}_{\text {DP }}\right), 128.57$

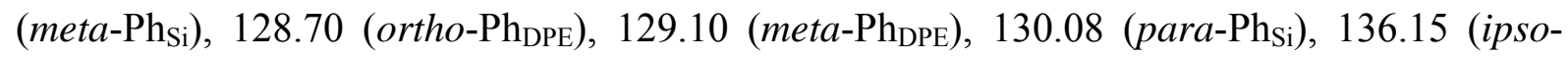
$\mathrm{Ph}_{\mathrm{Si}}$ ), 136.76 (ortho- $\mathrm{Ph}_{\mathrm{Si}}$ ), 148.03 (ipso- $\mathrm{Ph}_{\mathrm{DPE}}$ ) ppm.

${ }^{29} \mathrm{Si}\left\{{ }^{1} \mathrm{H}\right\}$ NMR (THF-d, $\left.79.5 \mathrm{MHz}\right): \delta-11.18\left(\mathrm{Ph}_{2} \mathrm{CDCH}_{2} \mathrm{SiPh}_{3}\right) \mathrm{ppm}$.

${ }^{2} \mathrm{H}$ NMR (THF- $\left.d_{8}, 61.1 \mathrm{MHz}\right): \delta 4.24$ (s, 1D) ppm.

Anal. Calcd for $\mathrm{C}_{32} \mathrm{H}_{27} \mathrm{DSi}$ (441.66 $\mathrm{g} \cdot \mathrm{mol}^{-1}$ ): C, 87.02; H, 6.62. Found: C, 86.61; H, 6.82\%. 
CI MS (100 eV) m/z: $364\left(\mathrm{M}^{+}-\mathrm{C}_{6} \mathrm{H}_{5}, 100 \%\right), 259$ (16); molecular peak could not be detected.

The resonance for the $\mathrm{Ph}_{2} \mathrm{CDCH}_{2} \mathrm{SiPh}_{3}$ carbon atom of (2,2'-diphenylethyl)triphenylsilane- $d_{1}$ expected at around $\delta 48 \mathrm{ppm}$ in the ${ }^{13} \mathrm{C}\left\{{ }^{1} \mathrm{H}\right\}$ NMR spectrum was not observed, probably due to low intensity as result of C,D coupling.

\section{${ }^{1} \mathrm{H},{ }^{13} \mathrm{C}\left\{{ }^{1} \mathrm{H}\right\}$ and ${ }^{29} \mathrm{Si}\left\{{ }^{1} \mathrm{H}\right\}$ NMR spectra of (2,2'-diphenylethyl)triphenylsilane-d $d_{1}\left(3-d_{1}\right)$}

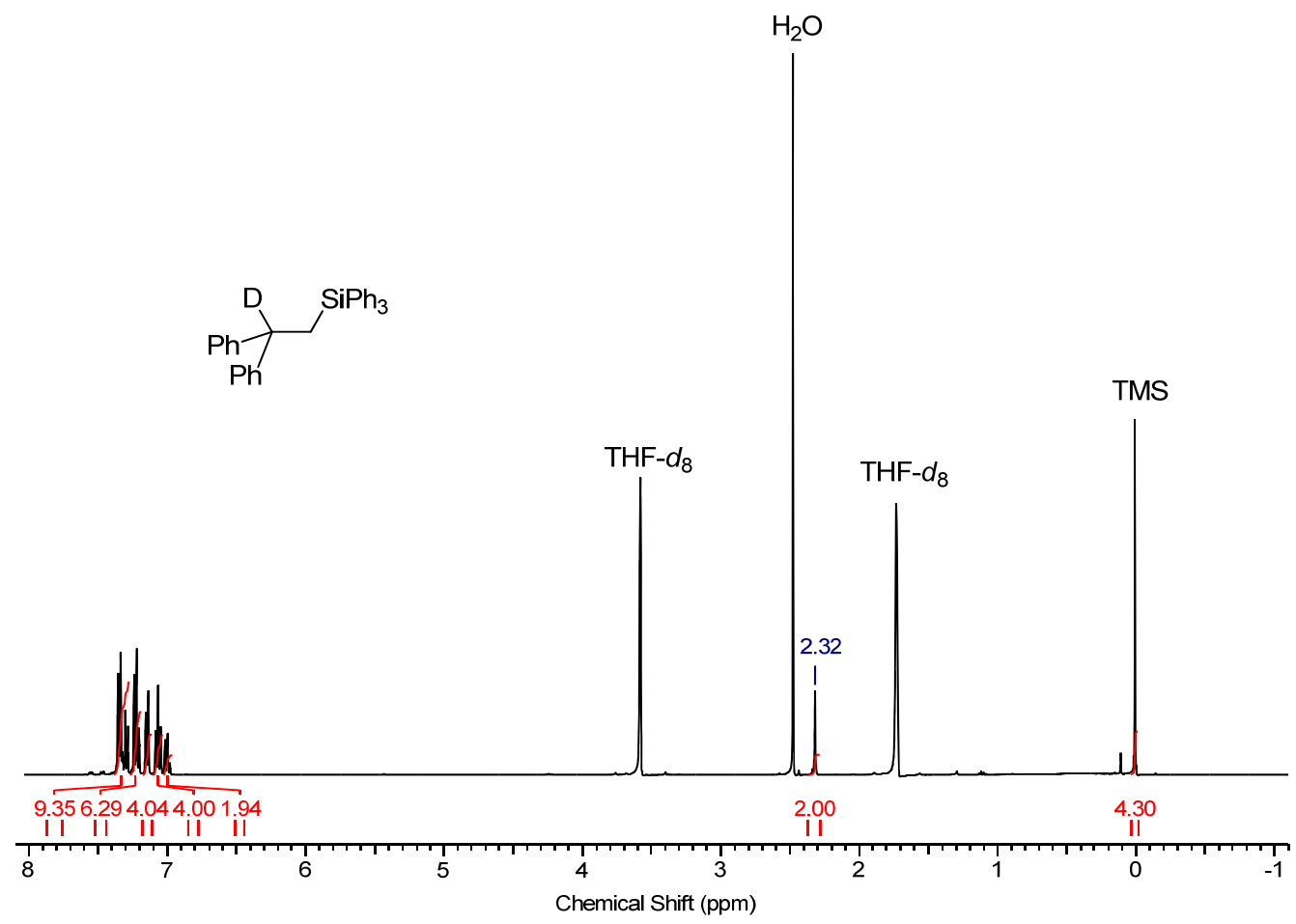

Figure S10. ${ }^{1} \mathrm{H}$ NMR spectrum of (2,2'-diphenylethyl)triphenylsilane- $d_{1}$ in THF- $d_{8}$ at $25{ }^{\circ} \mathrm{C}$. Residual $\mathrm{H}_{2} \mathrm{O}$ from THF- $d_{8}$. 


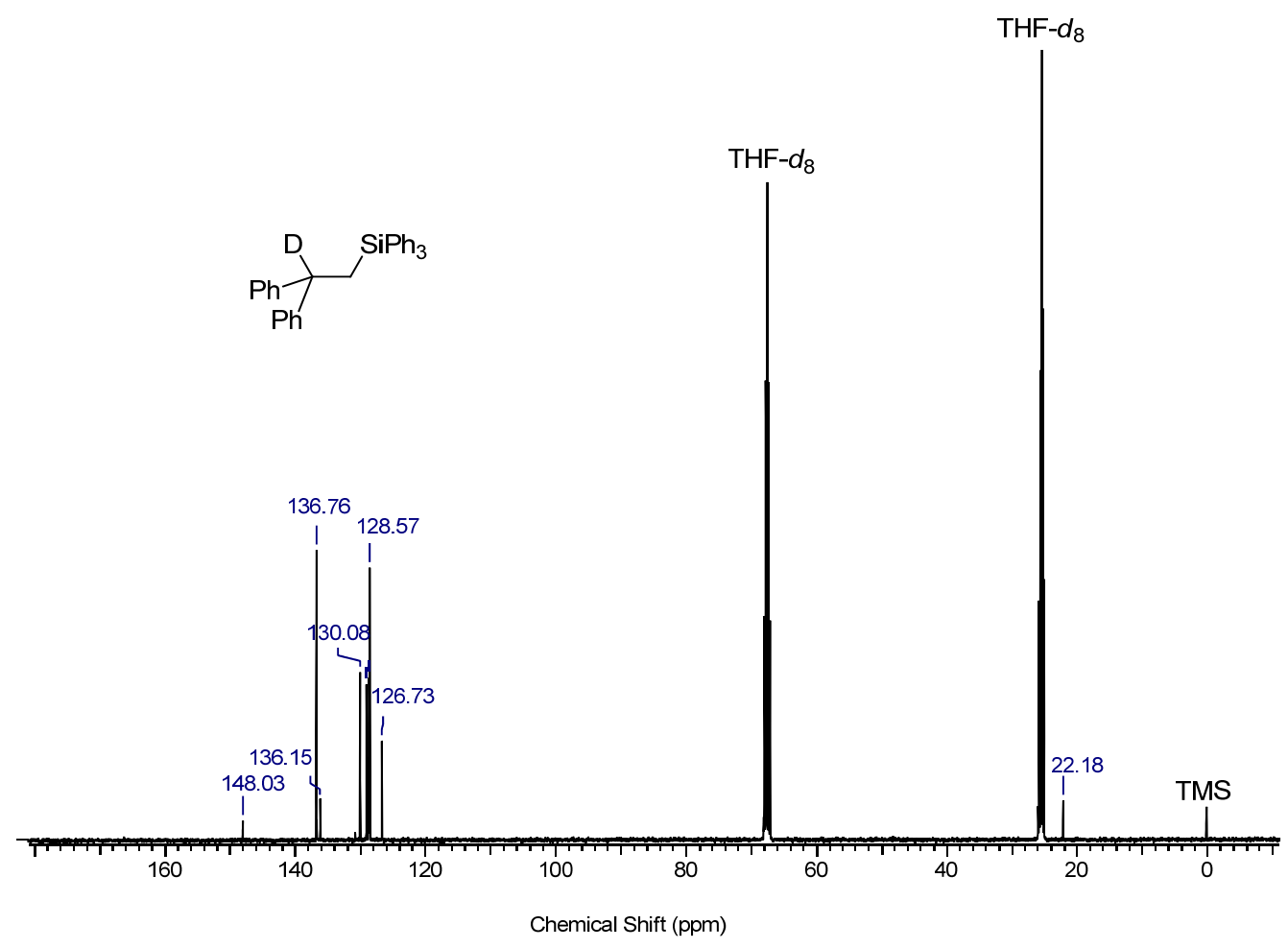

Figure S11. ${ }^{13} \mathrm{C}\left\{{ }^{1} \mathrm{H}\right\}$ NMR spectrum of (2,2'-diphenylethyl)triphenylsilane- $d_{1}$ in THF- $d_{8}$ at 25 ${ }^{\circ} \mathrm{C}$.

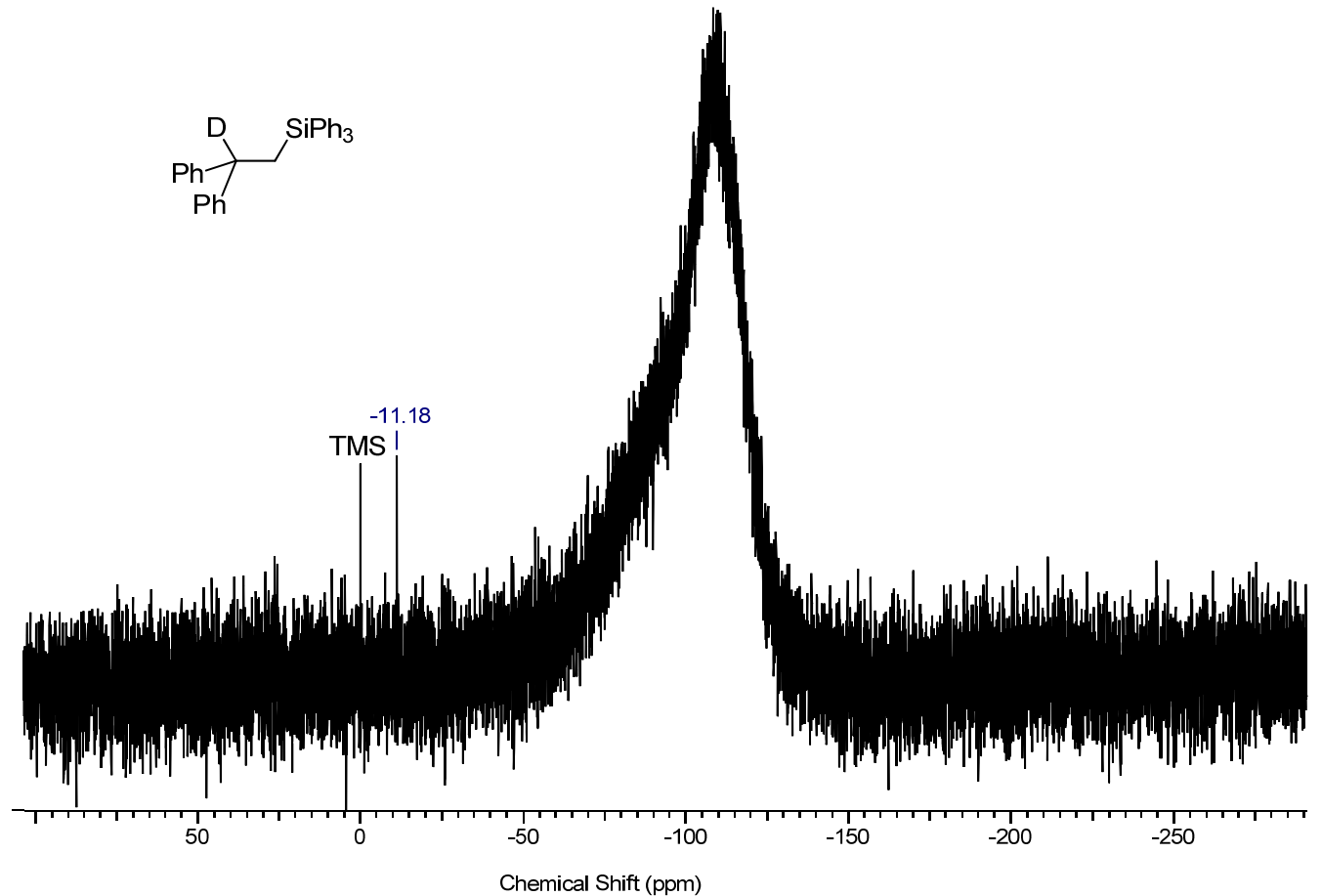

Figure $\mathrm{S} 12 .{ }^{29} \mathrm{Si}\left\{{ }^{1} \mathrm{H}\right\}$ NMR spectrum of $\left(2,2^{\prime}\right.$-diphenylethyl)triphenylsilane- $d_{1}$ in THF- $d_{8}$ at $25{ }^{\circ} \mathrm{C}$. 


\section{Hydrosilylation of alkenes}

Hydrosilylation experiments were performed as follows: A solution of catalyst $(2.5 \mu \mathrm{mol})$ in THF- $d_{8}(0.6 \mathrm{~mL})$ in a Young's NMR tube was treated with $1,1^{6}$-DPE $(0.10 \mathrm{mmol})$. Immediately the reaction mixture turned deep red. Silane $(0.11 \mathrm{mmol})$ was added and the reaction mixture was heated for the indicated period of time. The conversions of the substrates were determined by ${ }^{1} \mathrm{H}$ NMR spectroscopy. A typical workup was carried out as follows: the reaction mixture was quenched with water, extracted with $\mathrm{Et}_{2} \mathrm{O}$, dried over $\mathrm{Na}_{2} \mathrm{SO}_{4}$, filtered and the ether was removed under reduced pressure.

\section{Characterization of (1,2,2'-triphenylethyl)diphenylsilane}

The catalytic hydrosilylation reaction was performed as stated before. Triphenylethylene (25.6 mg, $0.10 \mathrm{mmol})$ and $\mathrm{Ph}_{2} \mathrm{SiH}_{2}(20 \mathrm{mg}, 00.11 \mathrm{mmol})$ were used as starting materials and the product was isolated without further purification and characterized via ${ }^{1} \mathrm{H},{ }^{13} \mathrm{C}\left\{{ }^{1} \mathrm{H}\right\}$ and ${ }^{29} \mathrm{Si}\left\{{ }^{1} \mathrm{H}\right\}$ NMR spectroscopy, CI-MS, and elemental analysis.

${ }^{1} \mathrm{H}$ NMR (THF- $\left.d_{8}, 400.1 \mathrm{MHz}\right): \delta 3.91\left(\mathrm{~d}, 1 \mathrm{H},{ }^{3} J_{\mathrm{H}, \mathrm{H}}=13.05 \mathrm{~Hz}, \mathrm{Ph}_{2} \mathrm{CHCHPhSiHPh}_{2}\right), 4.60$ $\left(\mathrm{d}, 1 \mathrm{H},{ }^{3} J_{\mathrm{H}, \mathrm{H}}=13.05 \mathrm{~Hz}, \mathrm{Ph}_{2} \mathrm{CHCHPhSiHPh}_{2}\right), 4.63\left(\mathrm{~s}, 1 \mathrm{H},{ }^{1} J_{\mathrm{Si}, \mathrm{H}}=100.14 \mathrm{~Hz}, \mathrm{SiH}\right), 6.81-$ 7.00 (m, 8H, Ph $\mathrm{DPE} / \mathrm{Si})$, 7.35-7.15 (m, 15H, Ph $\left.\mathrm{DPE}_{\mathrm{Di}}\right)$, 7.42-7.47 (m, 2H, $\left.\mathrm{Ph}_{\mathrm{DPE} / \mathrm{Si}}\right) \mathrm{ppm}$.

${ }^{13} \mathrm{C}\left\{{ }^{1} \mathrm{H}\right\} \quad \mathrm{NMR} \quad\left(\mathrm{THF}-d_{8}, \quad 100.6 \quad \mathrm{MHz}\right): \quad \delta \quad 40.52 \quad\left(\mathrm{Ph}_{2} \mathrm{CHCHPhSiHPh}\right), \quad 54.54$ $\left(\mathrm{Ph}_{2}\right.$ CHCHPhSiHPh $, 125.54\left(\mathrm{Ph}_{\mathrm{DPE} / \mathrm{Si}}\right), 126.18\left(\mathrm{Ph}_{\mathrm{DPE} / \mathrm{Si}}\right), 127.27\left(\mathrm{Ph}_{\mathrm{DPE} / \mathrm{Si}}\right), 128.27\left(\mathrm{Ph}_{\mathrm{DPE} / \mathrm{Si}}\right)$, $128.32\left(\mathrm{Ph}_{\mathrm{DPE} / \mathrm{Si}}\right), 128.38\left(\mathrm{Ph}_{\mathrm{DPE} / \mathrm{Si}}\right), 128.62\left(\mathrm{Ph}_{\mathrm{DPE} / \mathrm{Si}}\right), 128.95\left(\mathrm{Ph}_{\mathrm{DPE} / \mathrm{Si}}\right), 129.18\left(\mathrm{Ph}_{\mathrm{DPE} / \mathrm{Si}}\right)$, $129.61\left(\mathrm{Ph}_{\mathrm{DPE} / \mathrm{Si}}\right), 130.04\left(\mathrm{Ph}_{\mathrm{DPE} / \mathrm{Si}}\right), 130.28\left(\mathrm{Ph}_{\mathrm{DPE} / \mathrm{Si}}\right), 130.61\left(\mathrm{Ph}_{\mathrm{DPE} / \mathrm{Si}}\right), 130.82\left(\mathrm{Ph}_{\mathrm{DPE} / \mathrm{Si}}\right)$, 133.82 (ipso-Ph $\left.\mathrm{DPE}_{\mathrm{Si}}\right), 134.68$ (ipso-Ph $\left.\mathrm{DPE}_{\mathrm{DS}}\right), 136.45\left(\mathrm{Ph}_{\mathrm{DPE} / \mathrm{Si}}\right), 137.17\left(\mathrm{Ph}_{\mathrm{DPE} / \mathrm{Si}}\right), 141.66$ (ipso-Ph $\mathrm{DPE}_{\mathrm{DS}}$ ), 145.42 (ipso- $\mathrm{Ph}_{\mathrm{DPE} / \mathrm{Si}}$ ), 145.58 (ipso- $\left.\mathrm{Ph}_{\mathrm{DPE} / \mathrm{Si}}\right) \mathrm{ppm}$.

${ }^{29} \mathrm{Si}\left\{{ }^{1} \mathrm{H}\right\}$ NMR (THF-d, $\left.79.5 \mathrm{MHz}\right): \delta-12.92\left(\mathrm{Ph}_{2} \mathrm{CHCHPhSiHPh}\right) \mathrm{ppm}$.

Anal. Calcd for $\mathrm{C}_{32} \mathrm{H}_{28} \mathrm{Si}\left(440.66 \mathrm{~g} \cdot \mathrm{mol}^{-1}\right)$ : C, 87.22; H, 6.40. Found: C, 84.72; H, 6.45\%. CI MS (100 eV) m/z: $285\left(\mathrm{M}^{+}-2 \cdot \mathrm{C}_{6} \mathrm{H}_{5}-\mathrm{H}, 8 \%\right), 257$ (100), 256 (70), 181 (22), 179 (25), 167 (16); molecular peak could not be detected. 
${ }^{1} \mathrm{H},{ }^{13} \mathrm{C}\left\{{ }^{1} \mathrm{H}\right\}$ and ${ }^{29} \mathrm{Si}\left\{{ }^{1} \mathrm{H}\right\}$ NMR spectra of (1,2,2'-triphenylethyl)diphenylsilane

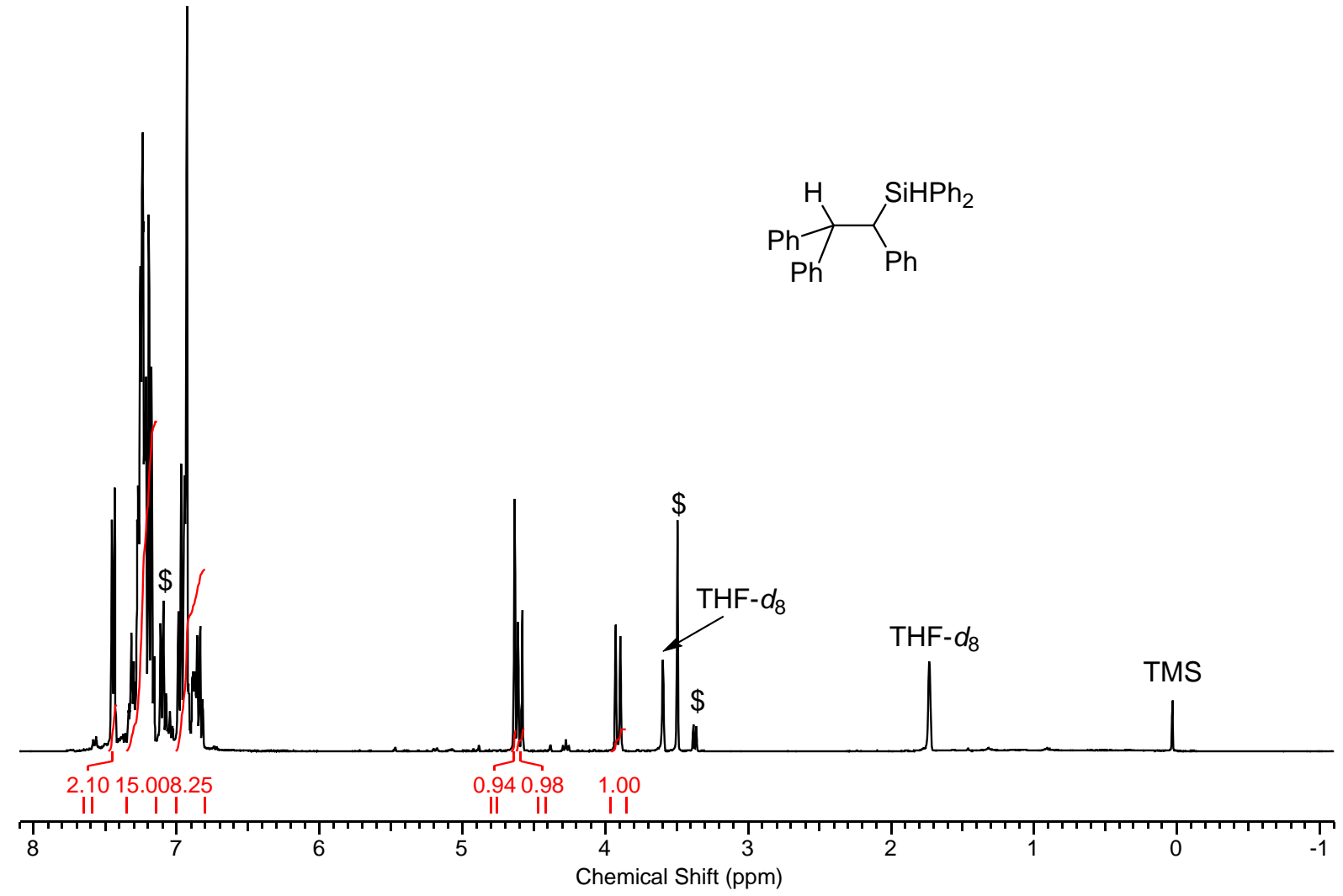

Figure S13. ${ }^{1} \mathrm{H}$ NMR spectrum of $\left(1,2,2^{\prime}\right.$-triphenylethyl)diphenylsilane in THF- $d_{8}$ at $25{ }^{\circ} \mathrm{C} . \$$ denotes an impurity. 


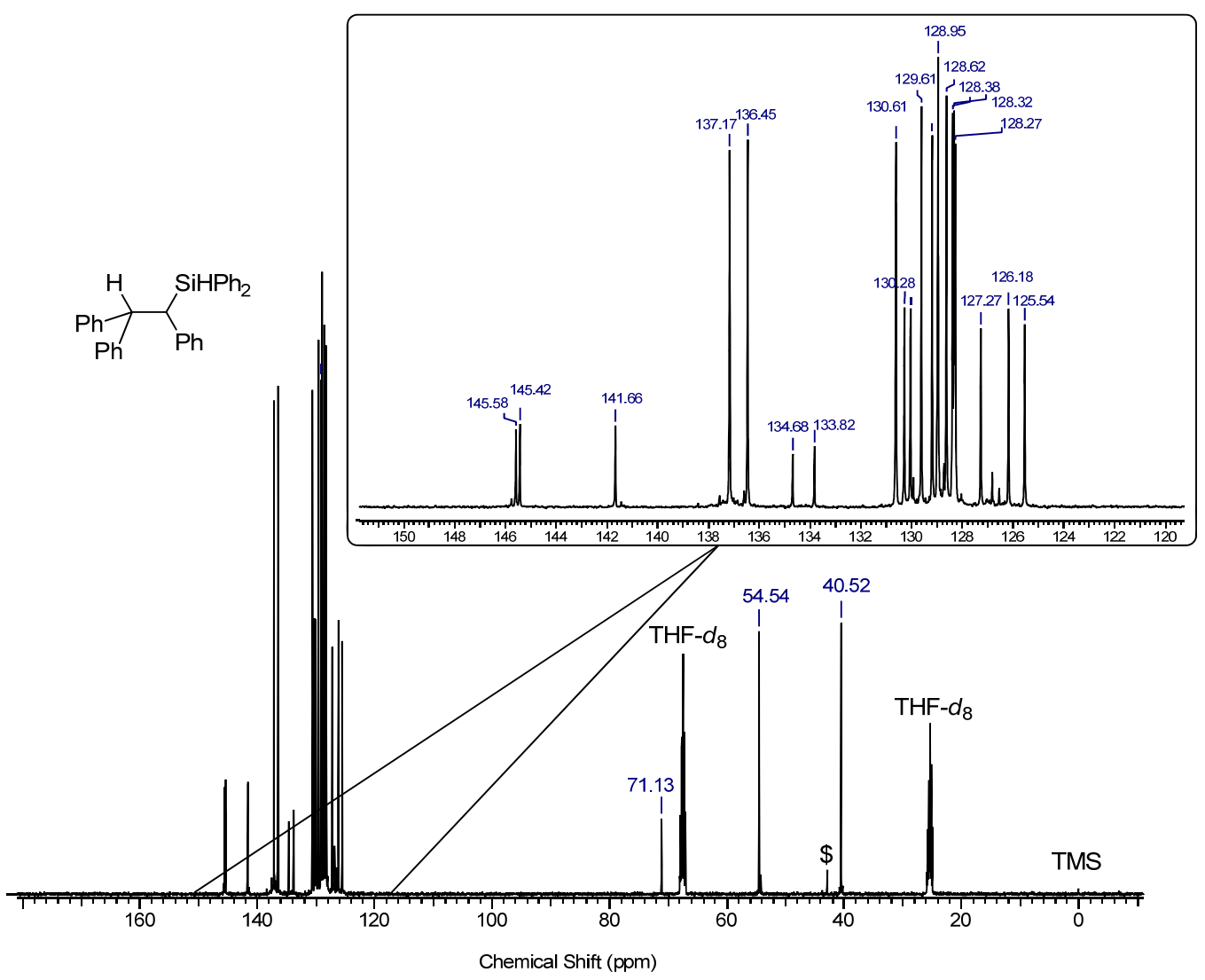

Figure S14. ${ }^{13} \mathrm{C}\left\{{ }^{1} \mathrm{H}\right\}$ NMR spectrum of $\left(1,2,2^{\prime}\right.$-triphenylethyl)diphenylsilane in THF- $d_{8}$ at 25 ${ }^{\circ} \mathrm{C}$. \$ denotes an impurity.

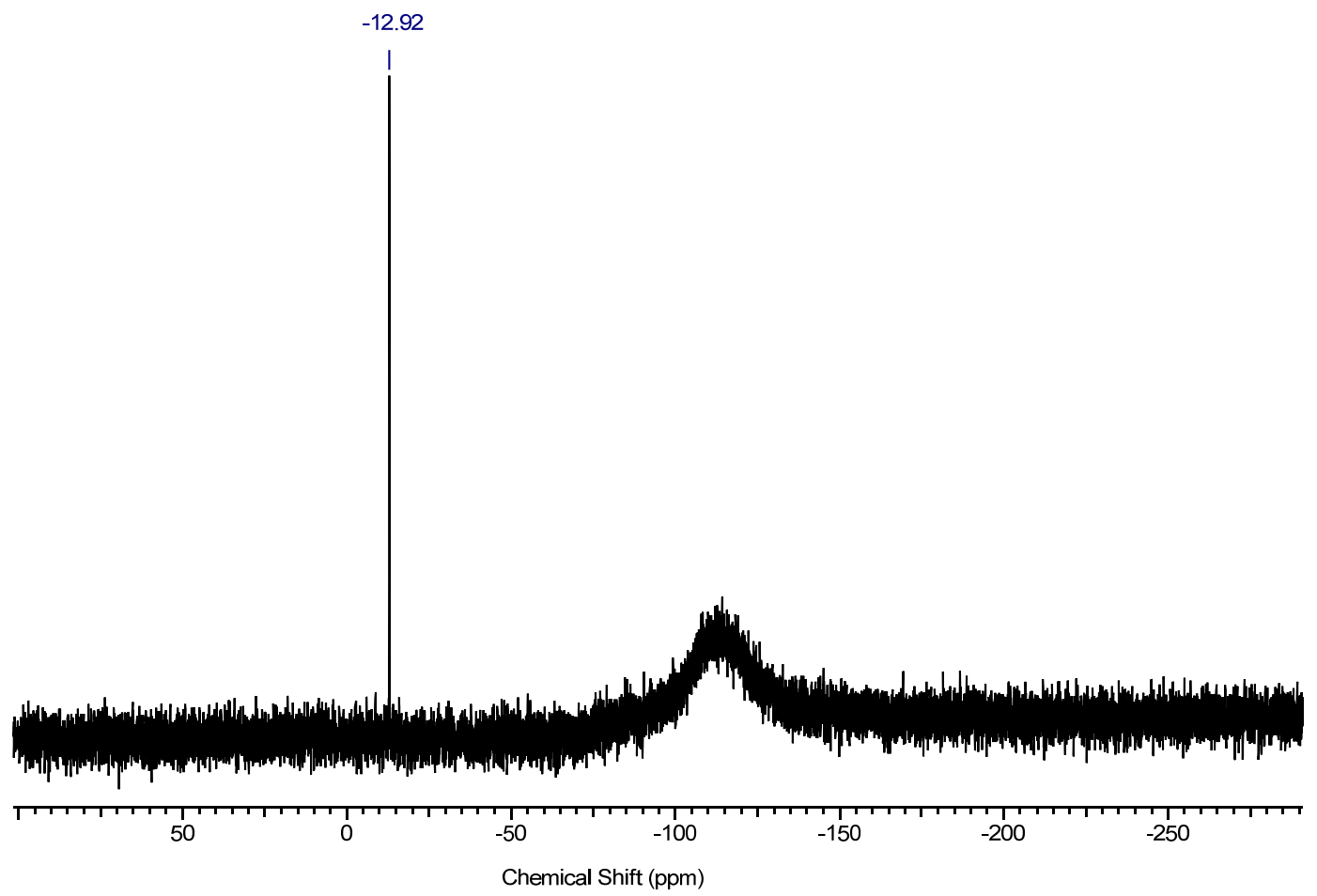

Figure S15. ${ }^{29} \mathrm{Si}\left\{{ }^{1} \mathrm{H}\right\}$ NMR spectrum of (1,2,2'-triphenylethyl)diphenylsilane in THF- $d_{8}$ at 25 ${ }^{\circ} \mathrm{C}$. 


\section{Characterization of (1,2,2'-triphenylethyl)phenylsilane}

The catalytic hydrosilylation reaction was performed as stated before. Triphenylethylene (25.6 mg, $0.10 \mathrm{mmol})$ and $\mathrm{PhSiH}_{3}(12 \mathrm{mg}, 00.11 \mathrm{mmol})$ were used as starting materials and the product was isolated without further purification, and characterized by ${ }^{1} \mathrm{H},{ }^{13} \mathrm{C}\left\{{ }^{1} \mathrm{H}\right\}$ and ${ }^{29} \mathrm{Si}\left\{{ }^{1} \mathrm{H}\right\}$ NMR spectroscopy, CI-MS and elemental analysis.

${ }^{1} \mathrm{H}$ NMR (THF-d $\left.d_{8}, 400.1 \mathrm{MHz}\right): \delta 3.68\left(\mathrm{ddd}, 1 \mathrm{H},{ }^{3} J_{\mathrm{H}, \mathrm{H}}=13.05 \mathrm{~Hz},{ }^{3} J_{\mathrm{H}, \mathrm{SiH}}=3.51 \mathrm{~Hz}\right.$, $\left.{ }^{3} J_{\mathrm{H}, \mathrm{SiH}}=1.76 \mathrm{~Hz}, \mathrm{Ph}_{2} \mathrm{CHCHPhSiH}_{2} \mathrm{Ph}\right), 4.08\left(\mathrm{dd}, 1 \mathrm{H},{ }^{1} J_{\mathrm{Si}, \mathrm{H}}=99.63 \mathrm{~Hz},{ }^{2} J_{\mathrm{SiH}, \mathrm{SiH}}=7.03 \mathrm{~Hz}\right.$, $\left.{ }^{3} J_{\mathrm{SiH}, \mathrm{H}}=1.76 \mathrm{~Hz}, \mathrm{SiH}\right), 4.19\left(\mathrm{dd}, 1 \mathrm{H},{ }^{1} J_{\mathrm{Si}, \mathrm{H}}=98.63 \mathrm{~Hz},{ }^{2} J_{\mathrm{SiH}, \mathrm{SiH}}=7.03 \mathrm{~Hz},{ }^{3} J_{\mathrm{SiH}, \mathrm{H}}=3.51 \mathrm{~Hz}\right.$, $\mathrm{SiH}), 4.60\left(\mathrm{~d}, 1 \mathrm{H},{ }^{3} J_{\mathrm{H}, \mathrm{H}}=13.05 \mathrm{~Hz}, \mathrm{Ph}_{2} \mathrm{CHCHPhSiH}_{2} \mathrm{Ph}\right), 6.85-6.95$ (m, 2H, $\mathrm{Ph}_{\mathrm{DPE} / \mathrm{Si}}$ ), 6.98$7.17\left(\mathrm{~m}, 11 \mathrm{H}, \mathrm{Ph}_{\mathrm{DPE} / \mathrm{Si}}\right), 7.22-7.31$ (m, 5H, $\left.\mathrm{Ph}_{\mathrm{DPE} / \mathrm{Si}}\right), 7.44-7.50\left(\mathrm{~m}, 2 \mathrm{H}, \mathrm{Ph}_{\mathrm{DPE} / \mathrm{Si}}\right) \mathrm{ppm}$.

${ }^{13} \mathrm{C}\left\{{ }^{1} \mathrm{H}\right\} \quad \mathrm{NMR} \quad\left(\mathrm{THF}-d_{8}, \quad 100.6 \quad \mathrm{MHz}\right): \quad \delta \quad 38.87 \quad\left(\mathrm{Ph}_{2} \mathrm{CHCHPhSiH}_{2} \mathrm{Ph}\right), \quad 54.20$ $\left(\mathrm{Ph}_{2}\right.$ CHCHPhSiH $\left.2 \mathrm{Ph}\right), 125.69\left(\mathrm{Ph}_{\mathrm{DPE} / \mathrm{Si}}\right), 126.36\left(\mathrm{Ph}_{\mathrm{DPE} / \mathrm{Si}}\right), 127.43\left(\mathrm{Ph}_{\mathrm{DPE} / \mathrm{Si}}\right), 128.25\left(\mathrm{Ph}_{\mathrm{DPE} / \mathrm{Si}}\right)$, $128.72\left(\mathrm{Ph}_{\mathrm{DPE} / \mathrm{Si}}\right), 128.87\left(\mathrm{Ph}_{\mathrm{DPE} / \mathrm{Si}}\right), 129.39\left(\mathrm{Ph}_{\mathrm{DPE} / \mathrm{Si}}\right), 129.70\left(\mathrm{Ph}_{\mathrm{DPE} / \mathrm{Si}}\right), 130.26\left(\mathrm{Ph}_{\mathrm{DPE} / \mathrm{Si}}\right)$, 132.05 (ipso-Ph $\left.\mathrm{DPP}_{\mathrm{DP}}\right), 136.76\left(\mathrm{Ph}_{\mathrm{DPE} / \mathrm{Si}}\right), 137.55$ ( $\left.\mathrm{Ph}_{\mathrm{DPE} / \mathrm{Si}}\right), 142.63$ (ipso- $\left.\mathrm{Ph}_{\mathrm{DPE} / \mathrm{Si}}\right), 145.28$ (ipso- $\mathrm{Ph}_{\mathrm{DPE} / \mathrm{Si}}$ ), 145.44 (ipso- $\left.\mathrm{Ph}_{\mathrm{DPE} / \mathrm{Si}}\right) \mathrm{ppm}$.

${ }^{29} \mathrm{Si}\left\{{ }^{1} \mathrm{H}\right\}$ NMR (THF-d, $\left.79.5 \mathrm{MHz}\right): \delta-24.97\left(\mathrm{Ph}_{2} \mathrm{CHCHPhSiH}_{2} \mathrm{Ph}\right) \mathrm{ppm}$.

One expected resonance of (1,2,2'-triphenylethyl)phenylsilane could not be detected in the ${ }^{13} \mathrm{C}\left\{{ }^{1} \mathrm{H}\right\}$ NMR spectrum.

Anal. Calcd for $\mathrm{C}_{26} \mathrm{H}_{24} \mathrm{Si}\left(364.56 \mathrm{~g} \cdot \mathrm{mol}^{-1}\right)$ : C, 85.66; H, 6.64. Found: C, 84.54; H, 6.78\%. CI MS (100 eV) m/z: 364 (M+ , 40\%), 363 (88), 287 (95), 257 (77), 181 (100), 167 (94). 


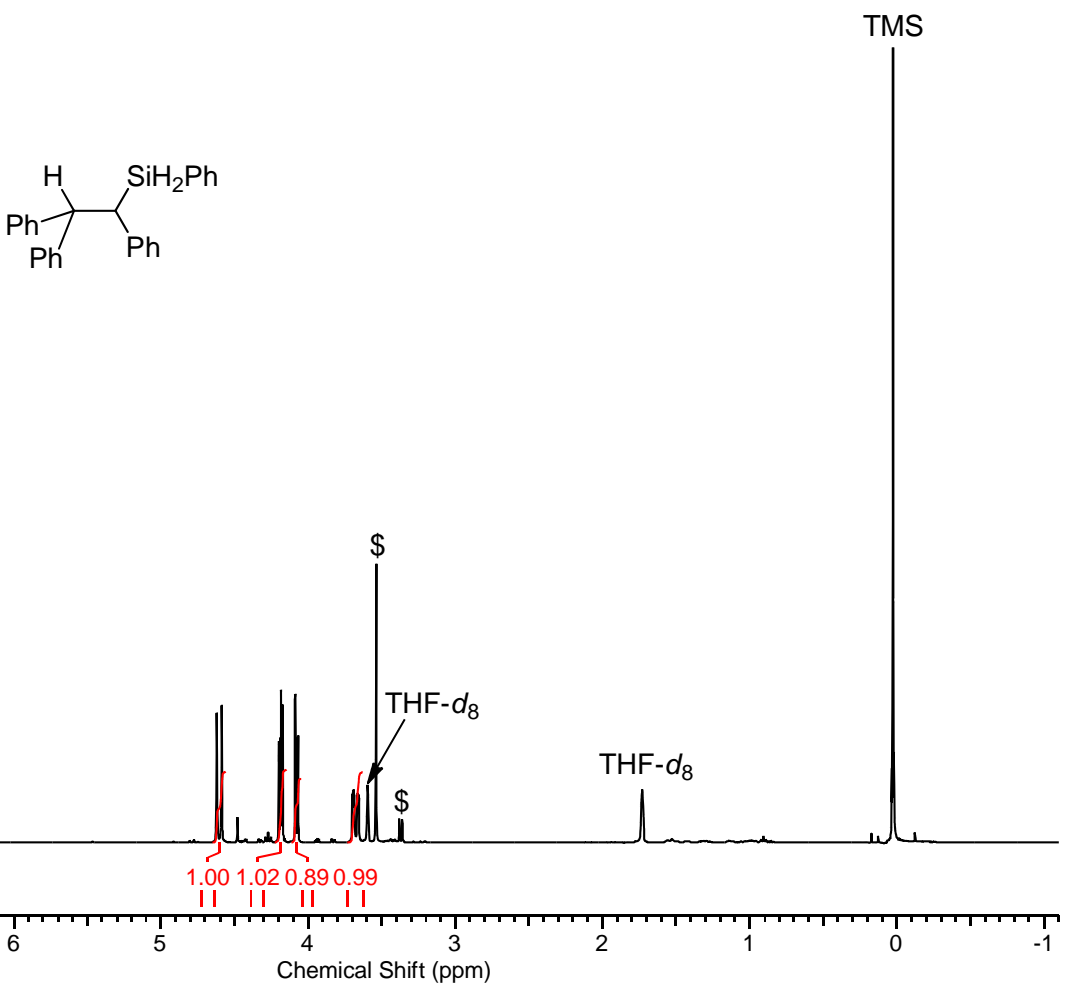

Figure S16. ${ }^{1} \mathrm{H}$ NMR spectrum of $\left(1,2,2^{\prime}\right.$-triphenylethyl)phenylsilane in THF- $d_{8}$ at $25{ }^{\circ} \mathrm{C} . \$$ denotes an impurity.

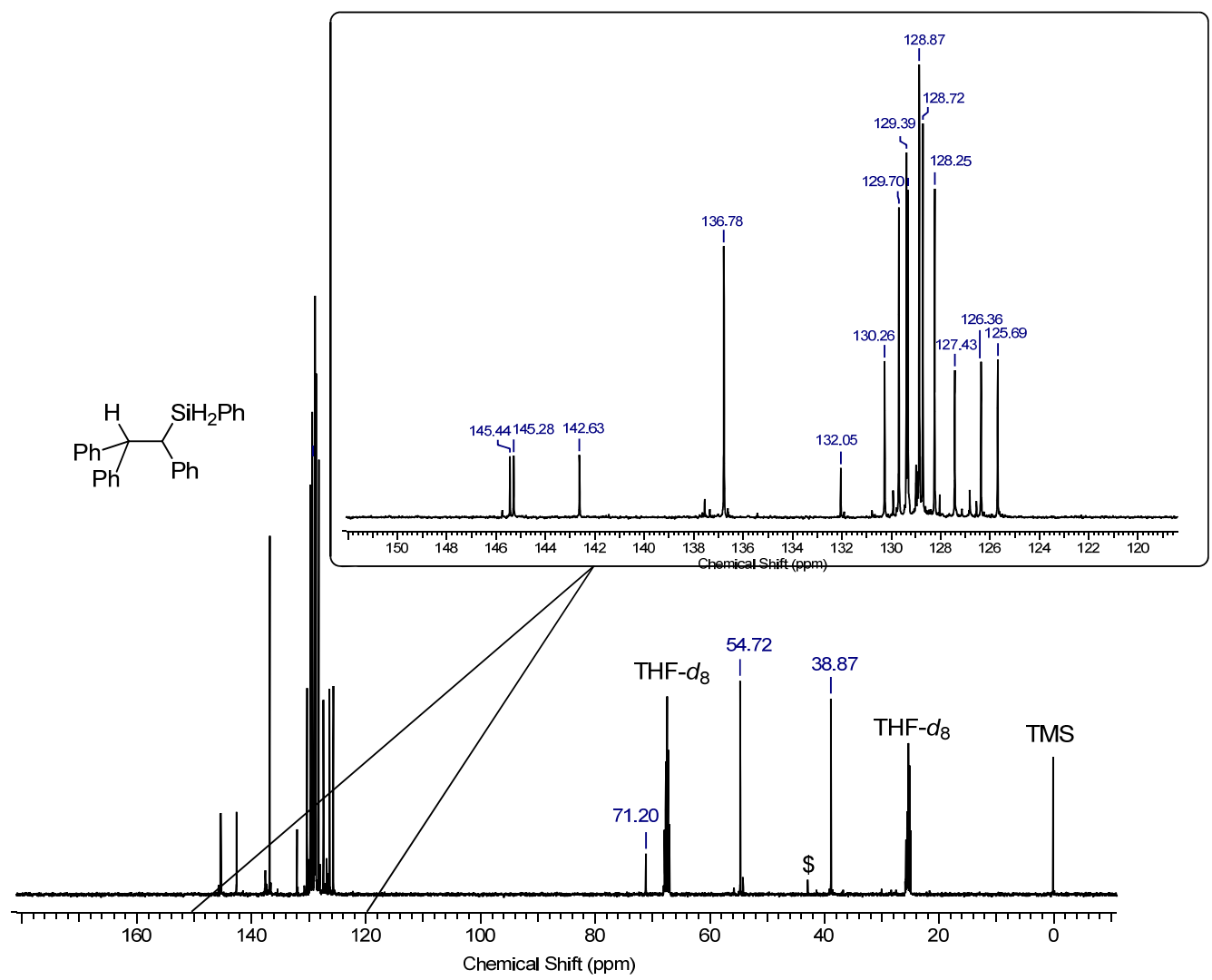

Figure S17. ${ }^{13} \mathrm{C}\left\{{ }^{1} \mathrm{H}\right\}$ NMR spectrum of (1,2,2'-triphenylethyl)phenylsilane in THF- $d_{8}$ at 25 ${ }^{\circ} \mathrm{C}$. $\$$ denotes an impurity. 


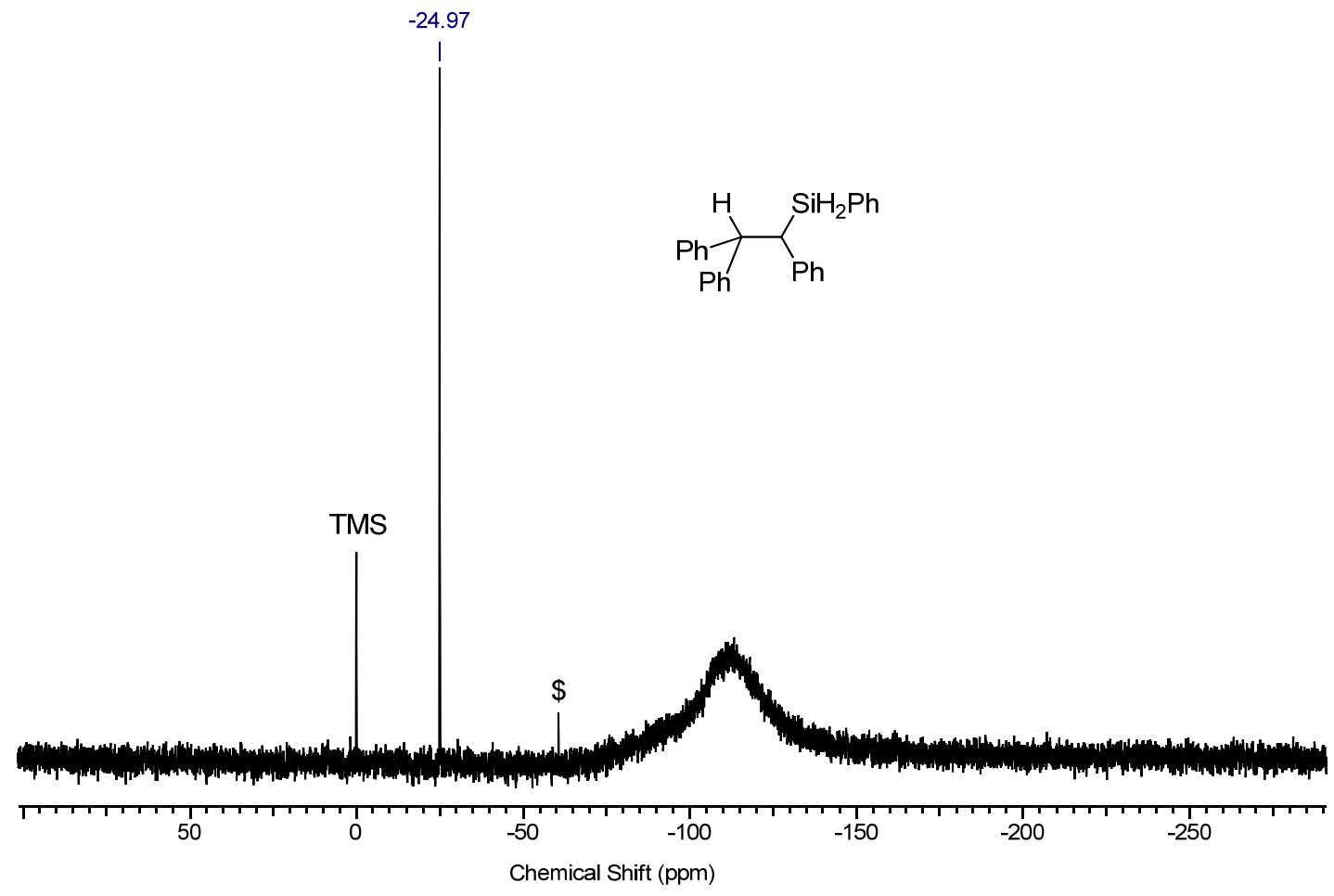

Figure S18. ${ }^{29} \mathrm{Si}\left\{{ }^{1} \mathrm{H}\right\}$ NMR spectrum of (1,2,2'-triphenylethyl)phenylsilane in THF- $d_{8}$ at 25 ${ }^{\circ} \mathrm{C} . \$$ denotes an impurity.

\section{in situ ${ }^{1} \mathrm{H}$ NMR Spectra of stoichiometric reactions}

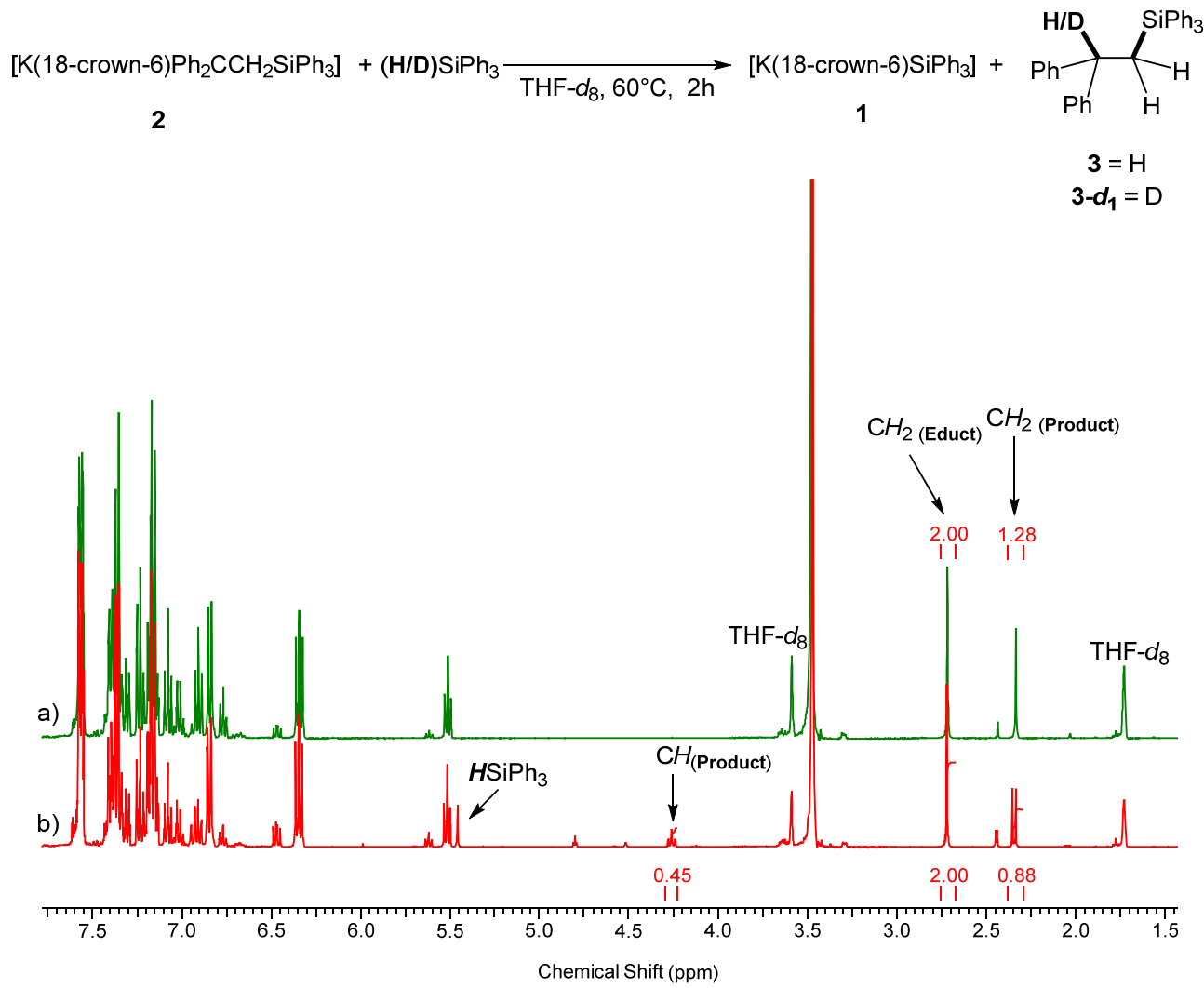

Figure S19. ${ }^{1} \mathrm{H}$ NMR Spectrum of the in situ reaction of [K(18-crown-6) $\left.\mathrm{Ph}_{2} \mathrm{CCH}_{2} \mathrm{SiPh}_{3}\right]$ with a) $\mathrm{DSiPh}_{3}$ or b) $\mathrm{HSiPh}_{3}$ in THF-d $d_{8}$ at $25^{\circ} \mathrm{C}$. 

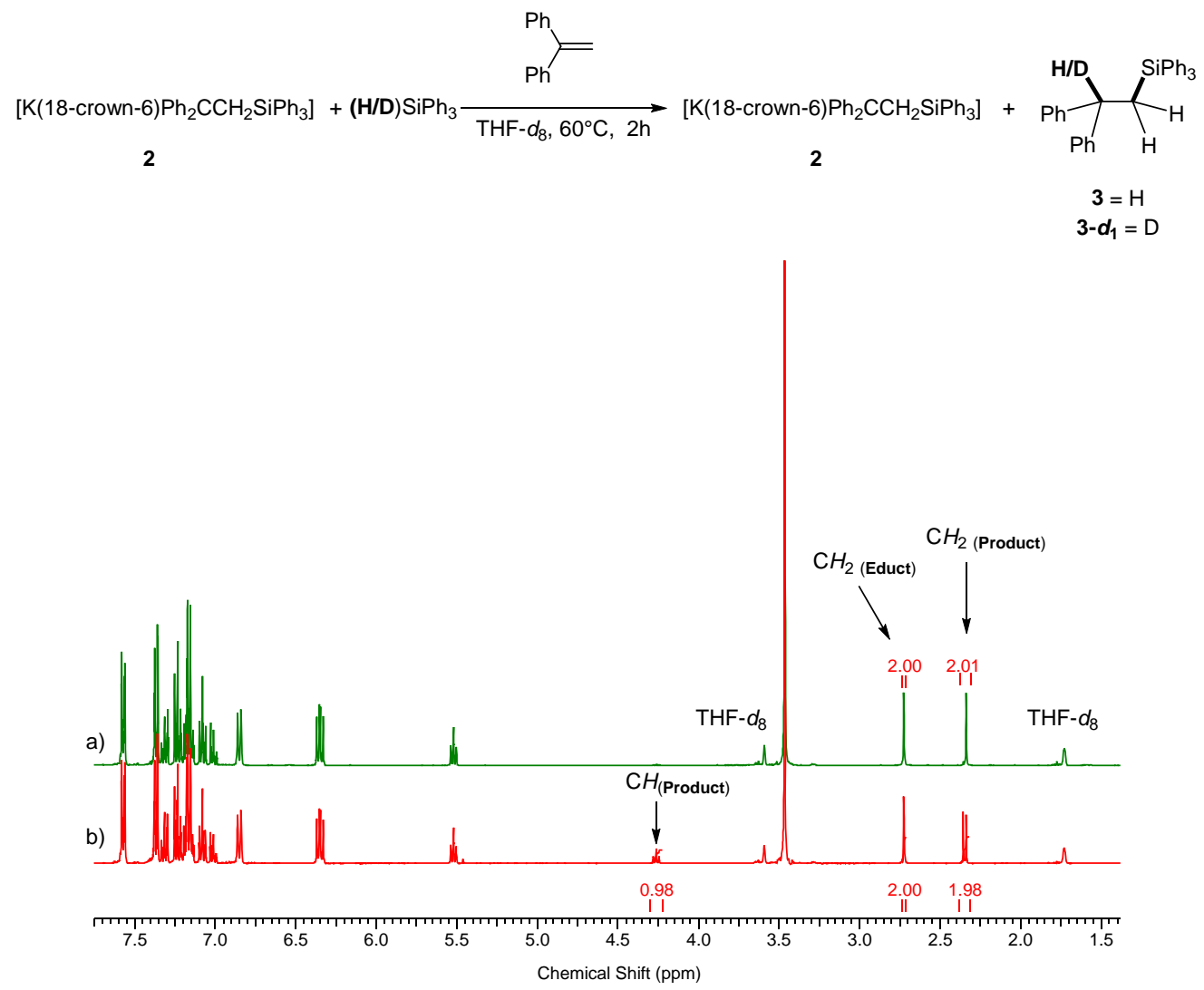

Figure S20. ${ }^{1} \mathrm{H}$ NMR Spectrum of the in situ reaction of [K(18-crown-6) $\left.\mathrm{Ph}_{2} \mathrm{CCH}_{2} \mathrm{SiPh}_{3}\right]$ with 1,1 $1^{\circ}$-DPE and a) $\mathrm{DSiPh}_{3}$ or b) $\mathrm{HSiPh}_{3}$ in THF-d $d_{8}$ at $25^{\circ} \mathrm{C}$.
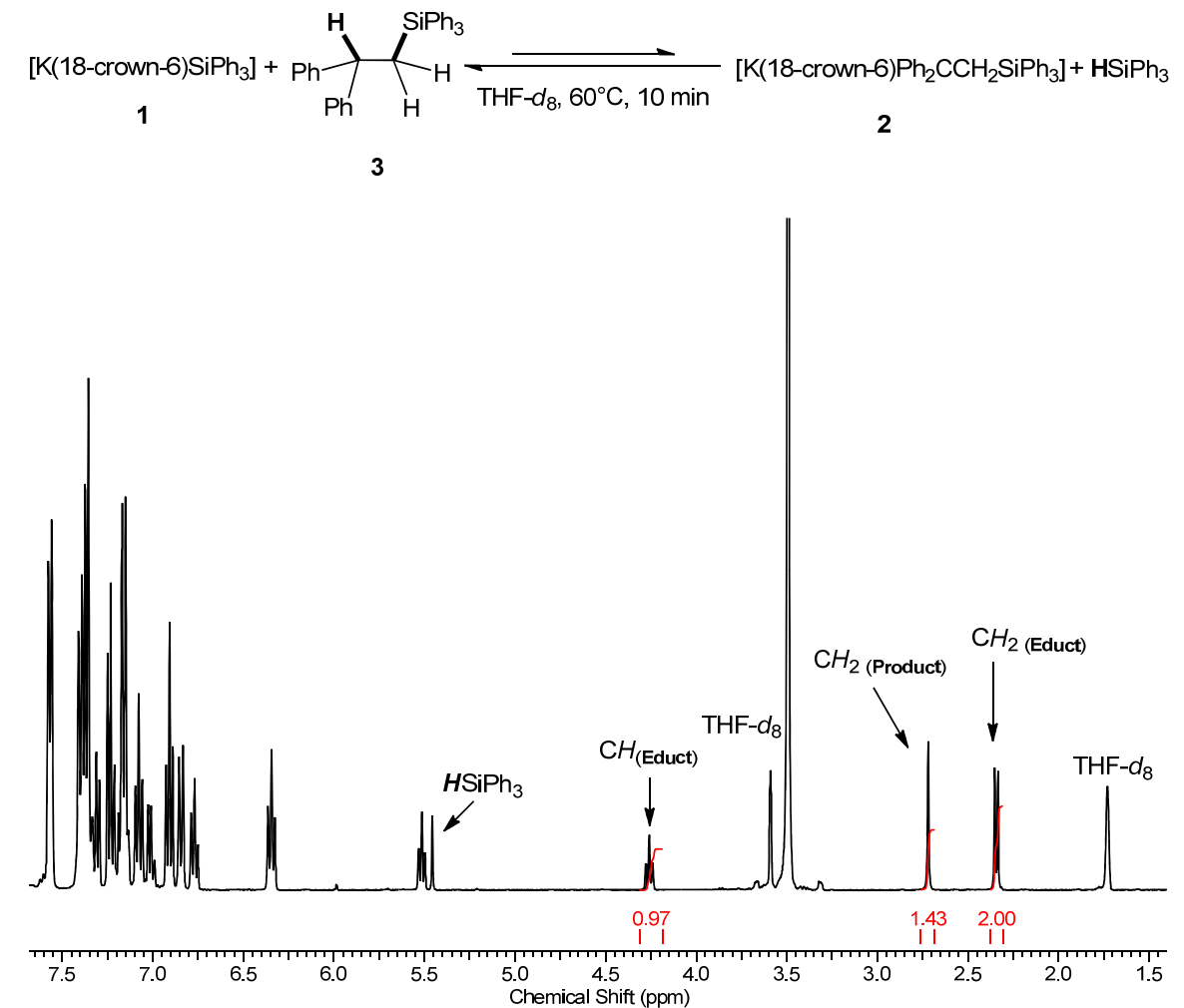

Figure S21. ${ }^{1} \mathrm{H}$ NMR Spectrum of the in situ reaction of $\left[\mathrm{K}\left(18\right.\right.$-crown-6) $\left.\mathrm{SiPh}_{3}\right]$ with $\left(2,2^{2}-\right.$ diphenylethyl)triphenylsilane in THF- $d_{8}$ at $25^{\circ} \mathrm{C}$. 


\section{Crystallography}

\section{Crystal structure determination of [K(18-crown-6) $\left.\mathrm{Ph}_{2} \mathrm{CCH}_{2} \mathrm{SiPh}_{3}\right]$ (2)}

X-ray diffraction data of a single crystal of 2 (red block, $0.19 \mathrm{~mm} \times 0.20 \mathrm{~mm} \times 0.23 \mathrm{~mm}$ ) were collected at $-173{ }^{\circ} \mathrm{C}$ on a Bruker D8 goniometer with an APEX CCD area-detector in $\omega$ scan mode. Mo- $\mathrm{K}_{\alpha}$ radiation (multilayer optics, $\lambda=0.71073 \AA$ ) from an Incoatec microsource was used. The SMART program package was used for the data collection and unit cell determination; processing of the raw frame data was performed using $\mathrm{SAINT}^{\mathrm{S} 5}$ the absorption correction was applied with MULABS. ${ }^{\mathrm{S} 6}$ The structure of $\mathrm{C}_{44} \mathrm{H}_{51} \mathrm{KO}_{6} \mathrm{Si}$ was solved in the monoclinic space group $P 22_{1} / c$ (no. 14) with $a=9.3694(7) \AA, b=24.0129(17)$ $\AA, c=19.4552(12) \AA, V=3902.6(5) \AA^{3}, Z=4, M=743.04 \mathrm{~g} \cdot \mathrm{mol}^{-1}$, and $\mu=0.215 \mathrm{~mm}^{-1}$ by direct methods using SIR-92. ${ }^{\mathrm{S} 7}$ The refinement was carried out with anisotropic displacement parameters for all non-hydrogen against $F^{2}$ with SHELXL-2013 ${ }^{\mathrm{S} 8}$ as implemented in the WinGX $^{\mathrm{S9}}$ program system. Convergence was reached with $R 1=0.0393, w R 2=0.0775(I>$ $2 \sigma(I))$ and $R 1=0.0592, w R 2=0.0840$ (all data), $G o F=0.907$. The program Diamond was used for the graphical representation. ${ }^{\mathrm{S} 10}$ CCDC reference number 1454773 , these data can be obtained free of charge from the Cambridge Crystallographic Data Centre via $\underline{\text { www.ccdc.cam.ac.uk/data_request/cif. }}$

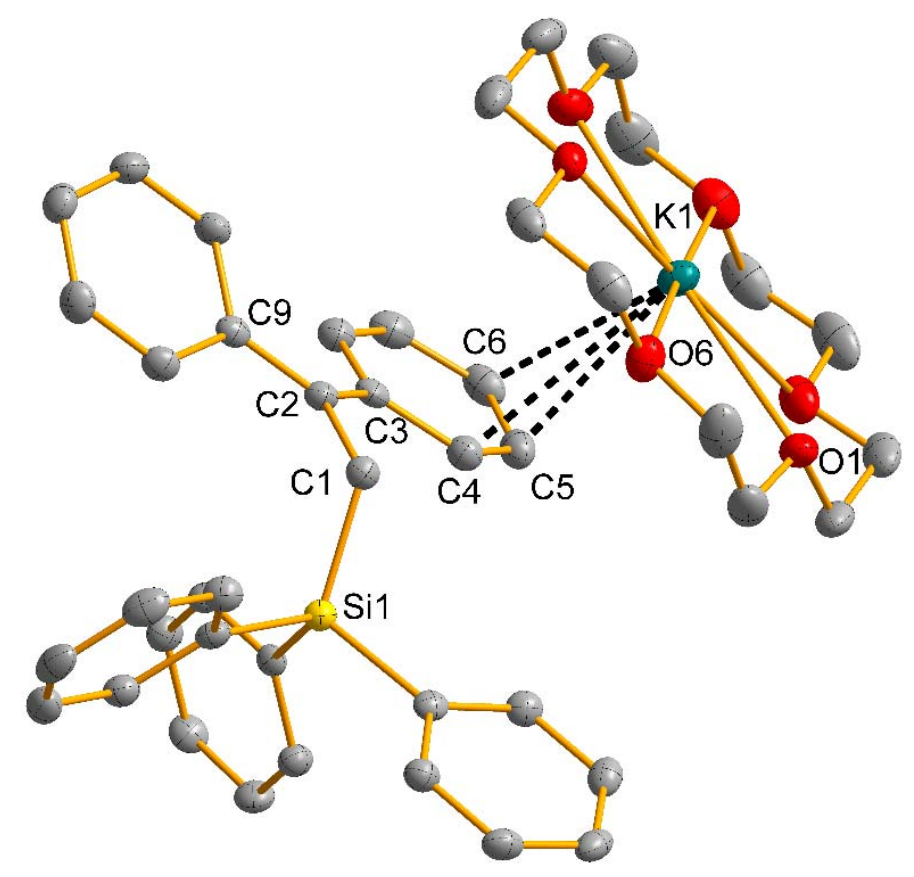

Figure S22. Molecular structure of 2 (cutout). Displacement ellipsoids are shown at the 50\% probability level; hydrogen atoms are omitted for clarity. 


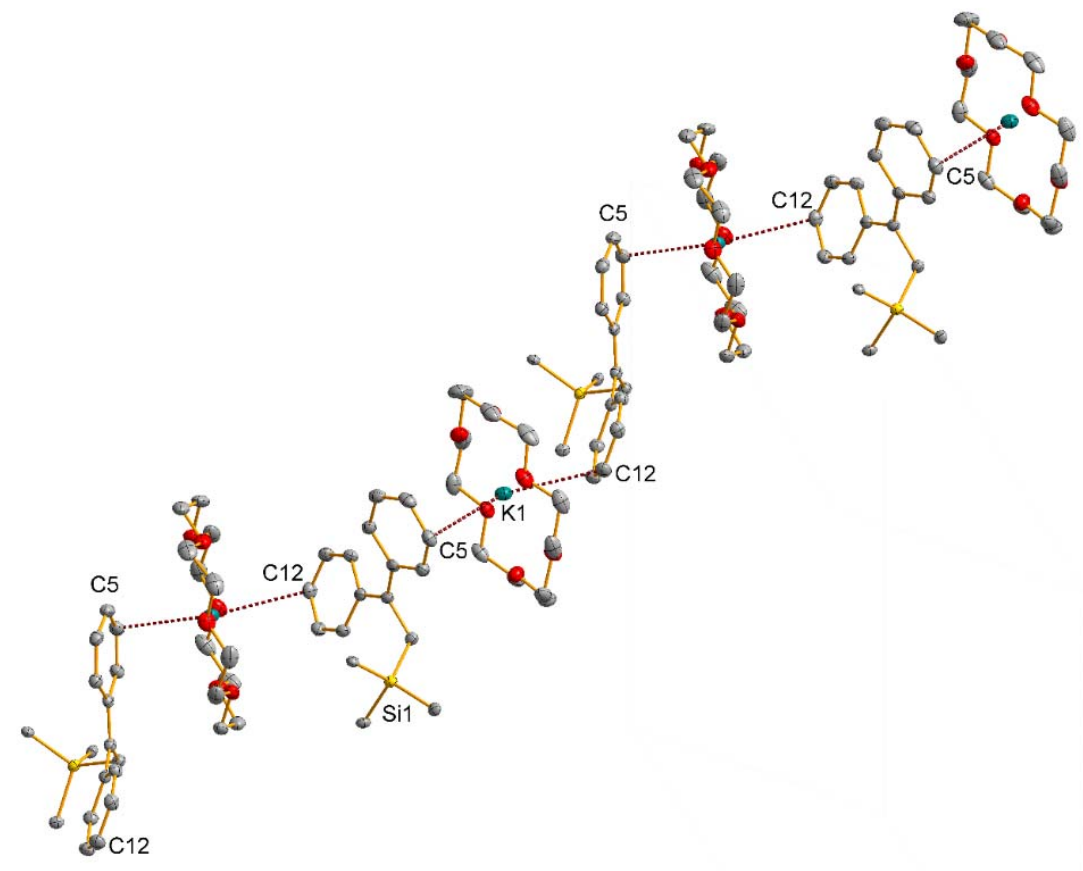

Figure S23. Molecular structure of 2 (extended structure). Displacement ellipsoids are shown at the 50\% probability level; hydrogen atoms and phenyl rings at the Sil atom are omitted for clarity.

\section{Kinetic studies}

A typical kinetic experiment was performed as follows. In a glovebox a solution of catalyst in THF- $d_{8}(0.1 \mathrm{~mL})$ in a vial was treated with a solution of $1,1^{6}$-DPE in THF- $d_{8}(0.2 \mathrm{~mL})$. Immediately the reaction mixture turned deep red and a solution of $\mathrm{HSiPh}_{3}$ in THF-d $(0.1$ $\mathrm{mL}$ ) was added. The reaction solution was then transferred to a J. Young type NMR tube, the vial was flushed twice with THF- $\mathrm{d}_{8}(2 \times 0.1 \mathrm{~mL})$ and a solution of hexamethylbenzene $(0.1$ $\mathrm{mL}, 33.3 \mu \mathrm{mol})$ in THF- $d_{8}$ as internal standard was added to the NMR tube. The reaction mixture was heated at $60{ }^{\circ} \mathrm{C}$ for the indicated period of time and the NMR tube was cooled with an dry ice / ethnaol mixture subsequently. The conversions of the substrates were determined by ${ }^{1} \mathrm{H}$ NMR spectroscopy. 


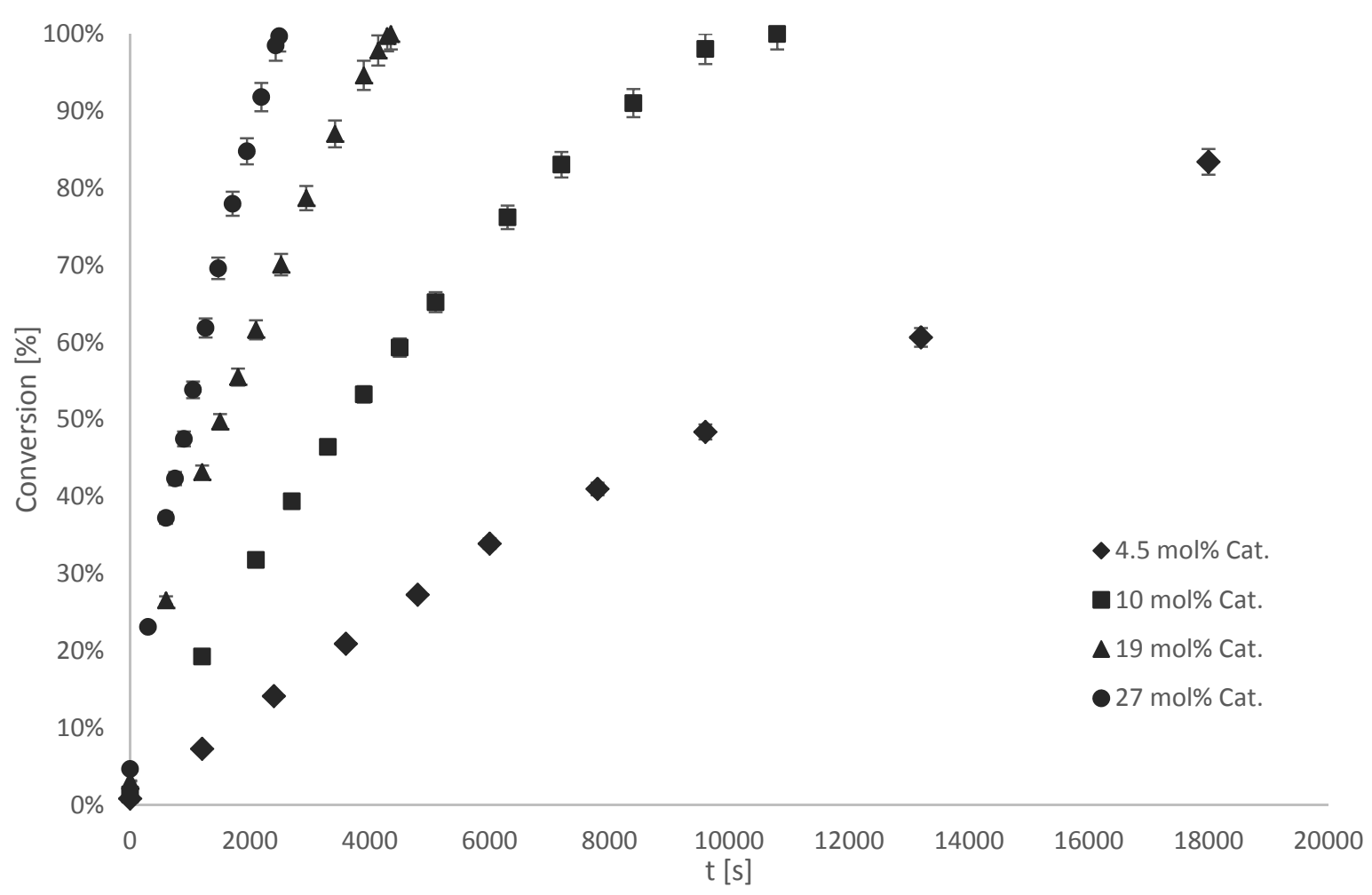

Figure S24. Time conversion plot (only formation of the hydrosilylation product) of the hydrosilylation of 1,1-DPE with $\mathrm{HSiPh}_{3}$ with different catalyst concentrations.

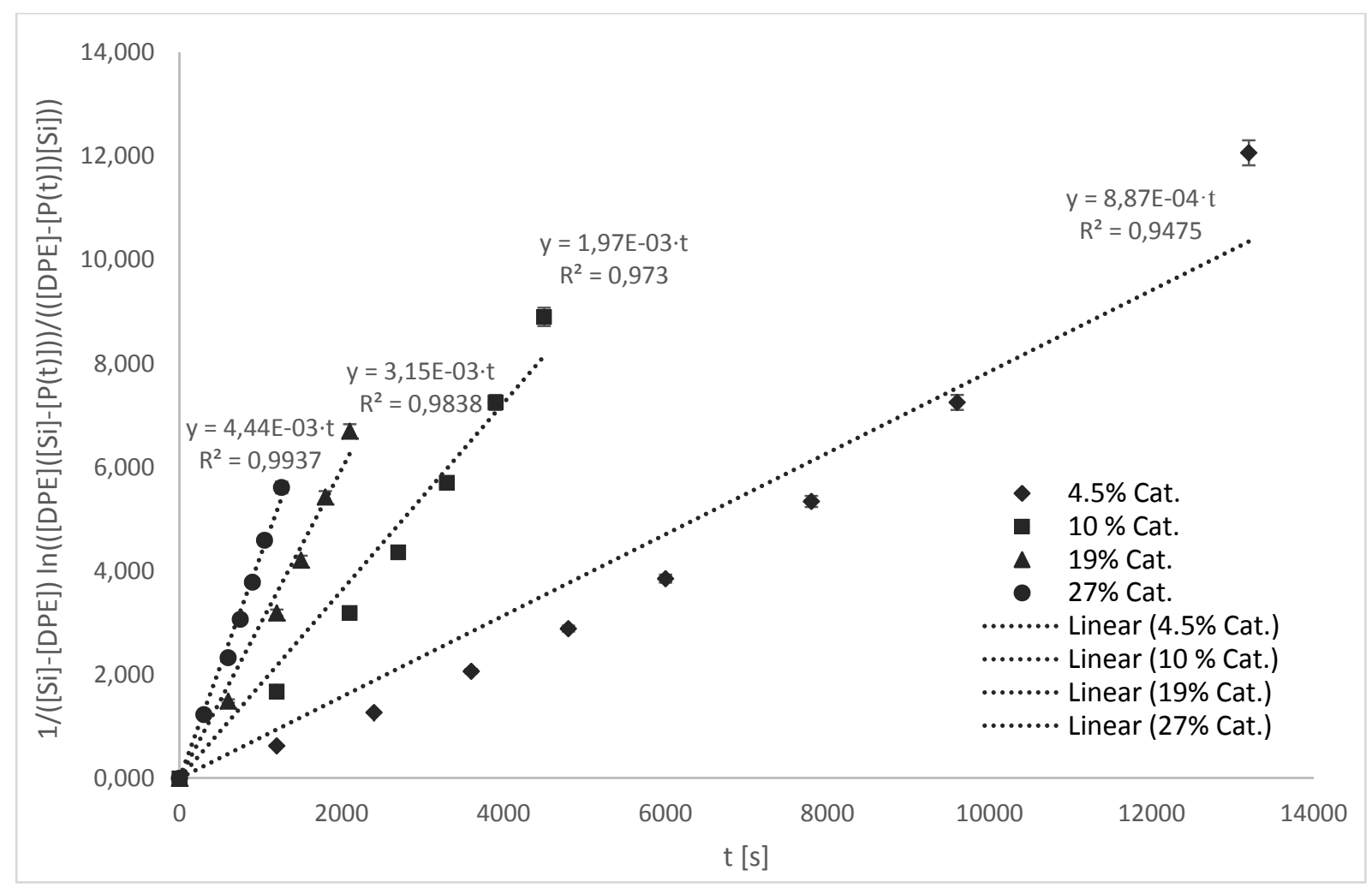

Figure S25. Semi-logarithmic plot of product formation (up to $60 \%$ conversion) with different catalyst concentrations. 


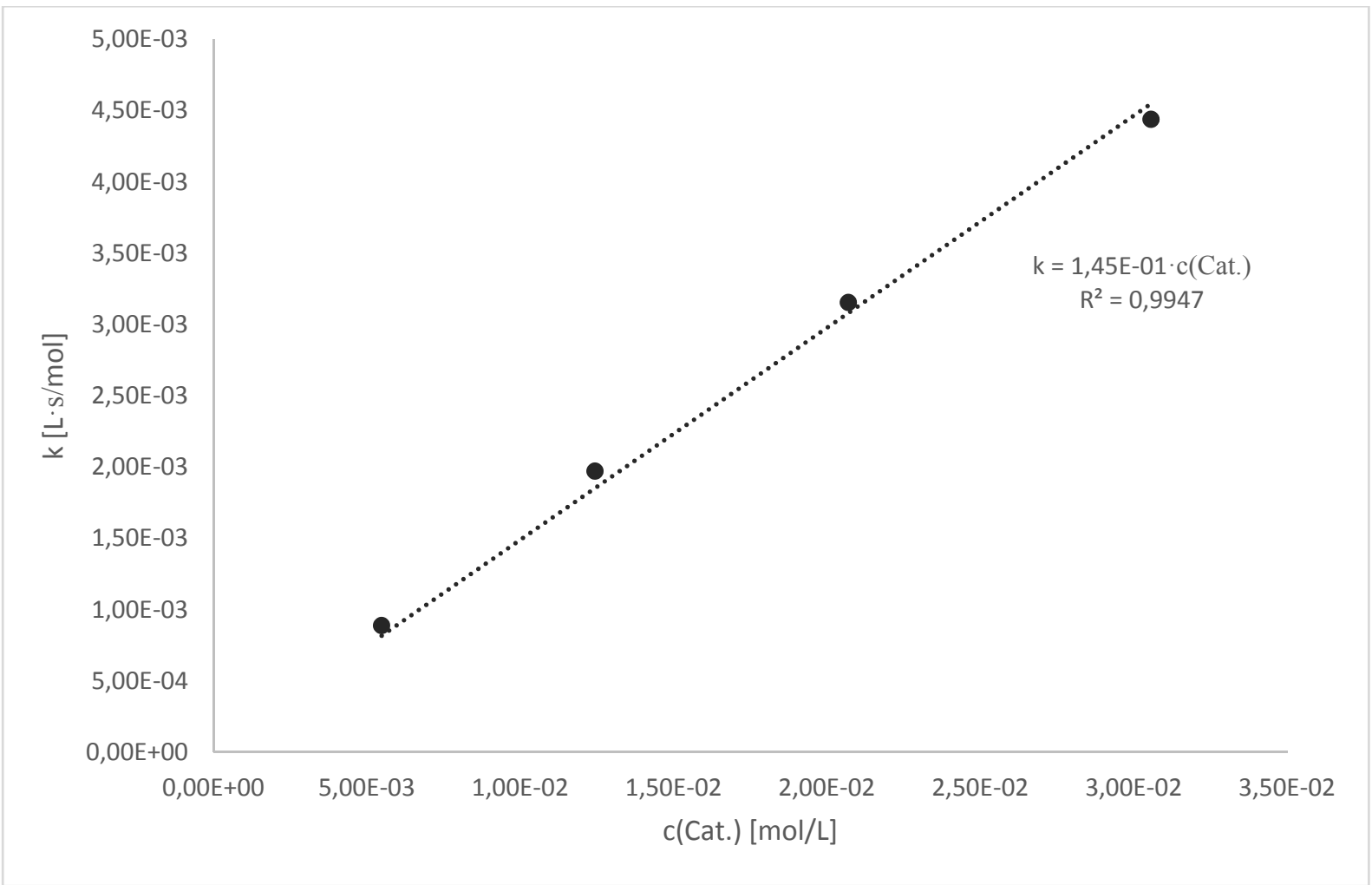

Figure S26. Rate constants $k$ versus catalyst concentration.

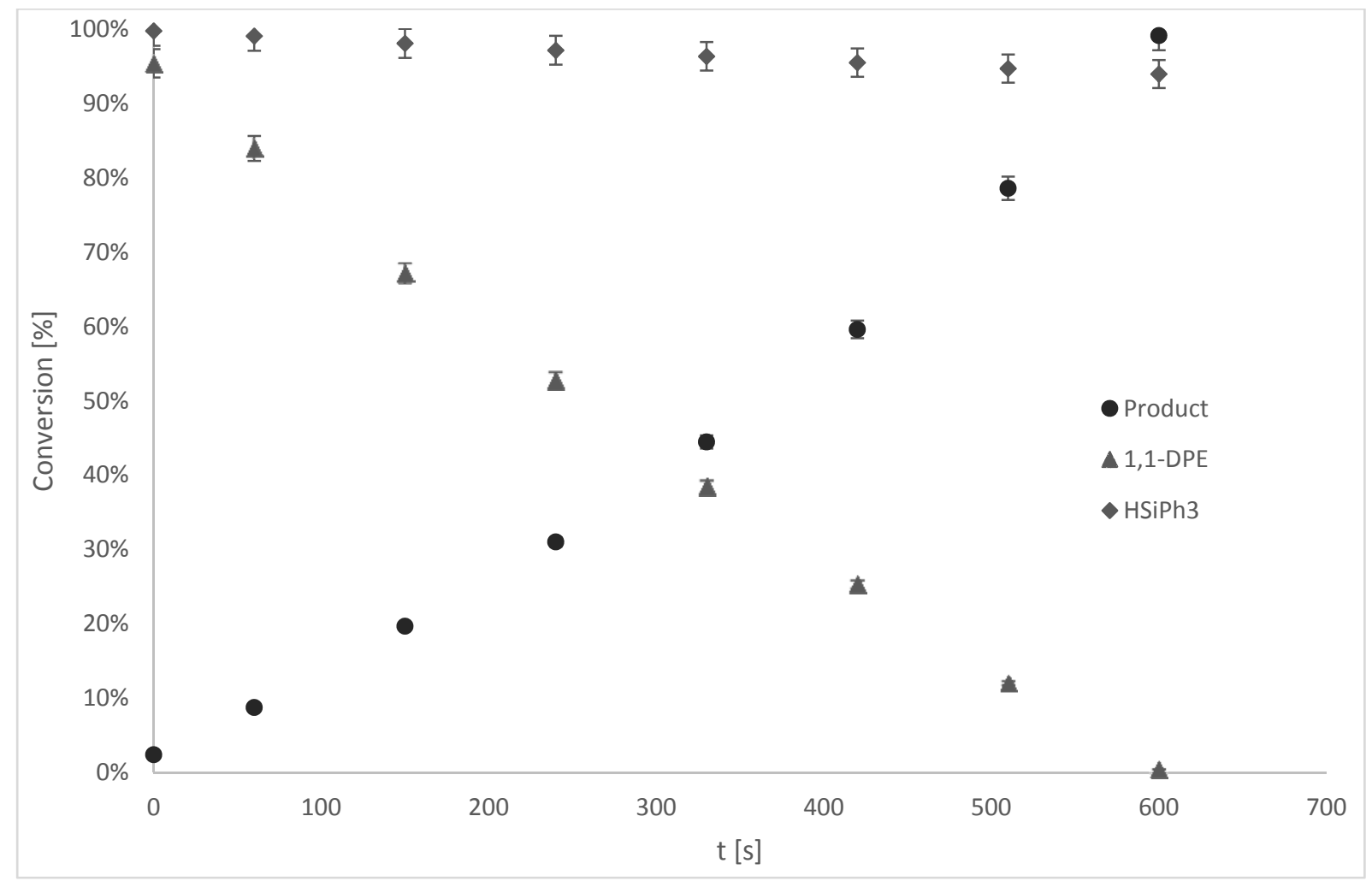

Figure S27. Time conversion plot of the hydrosilylation of 1,1-DPE with $\mathrm{HSiPh}_{3}$ (excess). 


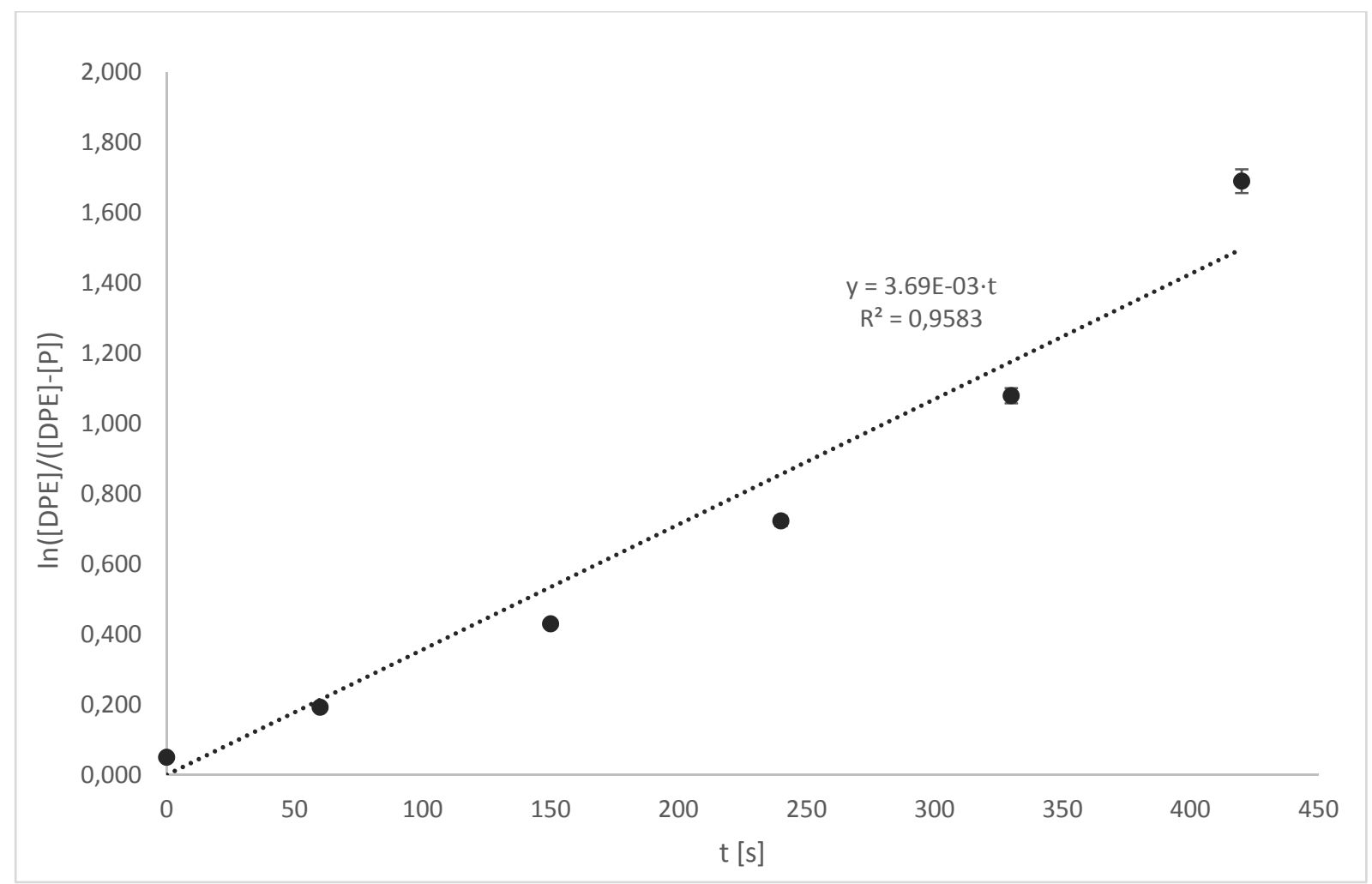

Figure S28. Logarithmic plot of product formation (up to $60 \%$ conversion) with excess of $\mathrm{HSiPh}_{3}$.

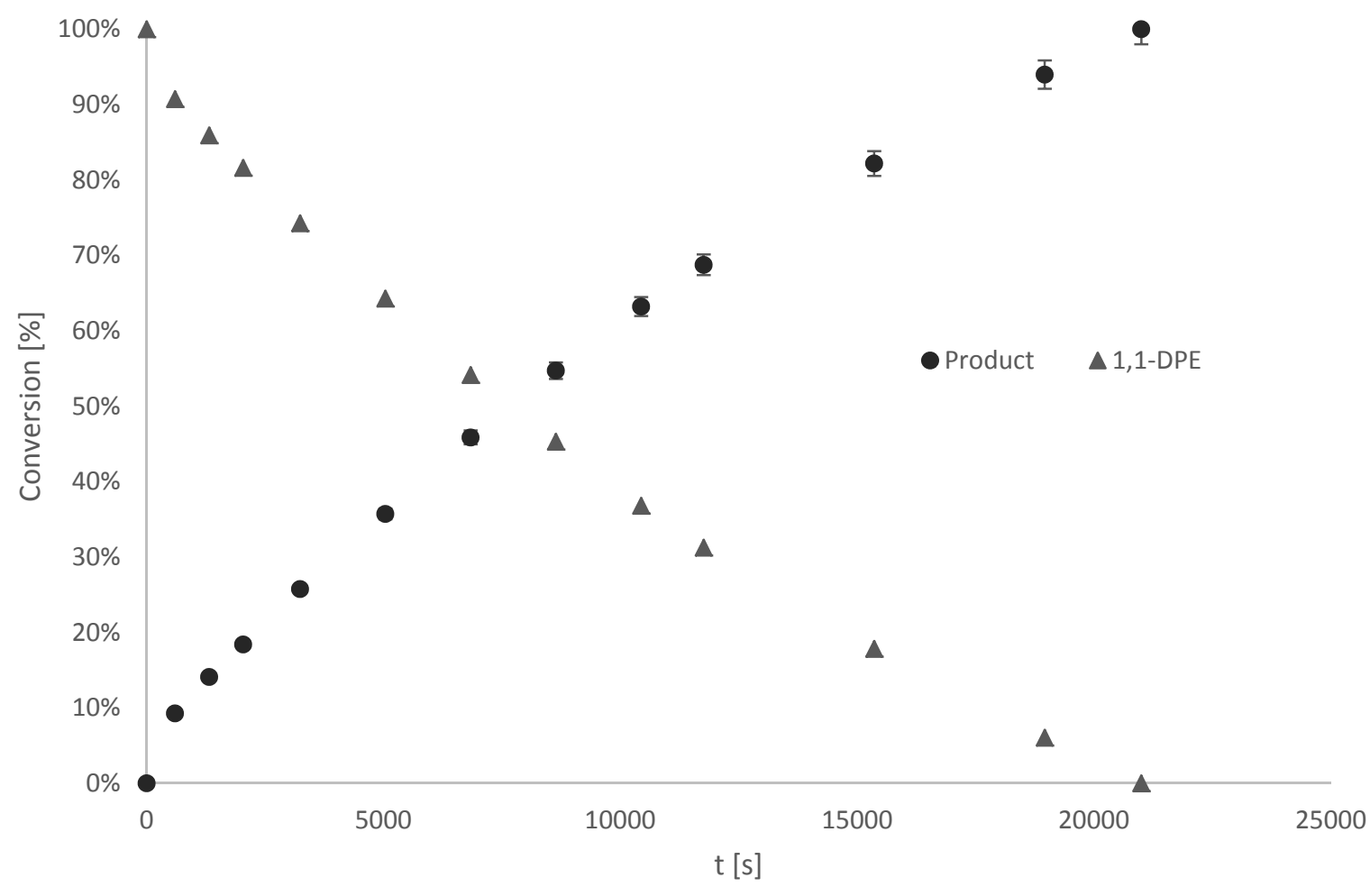

Figure S29. Time conversion plot of the hydrosilylation of 1,1-DPE with $\mathrm{DSiPh}_{3}$. 


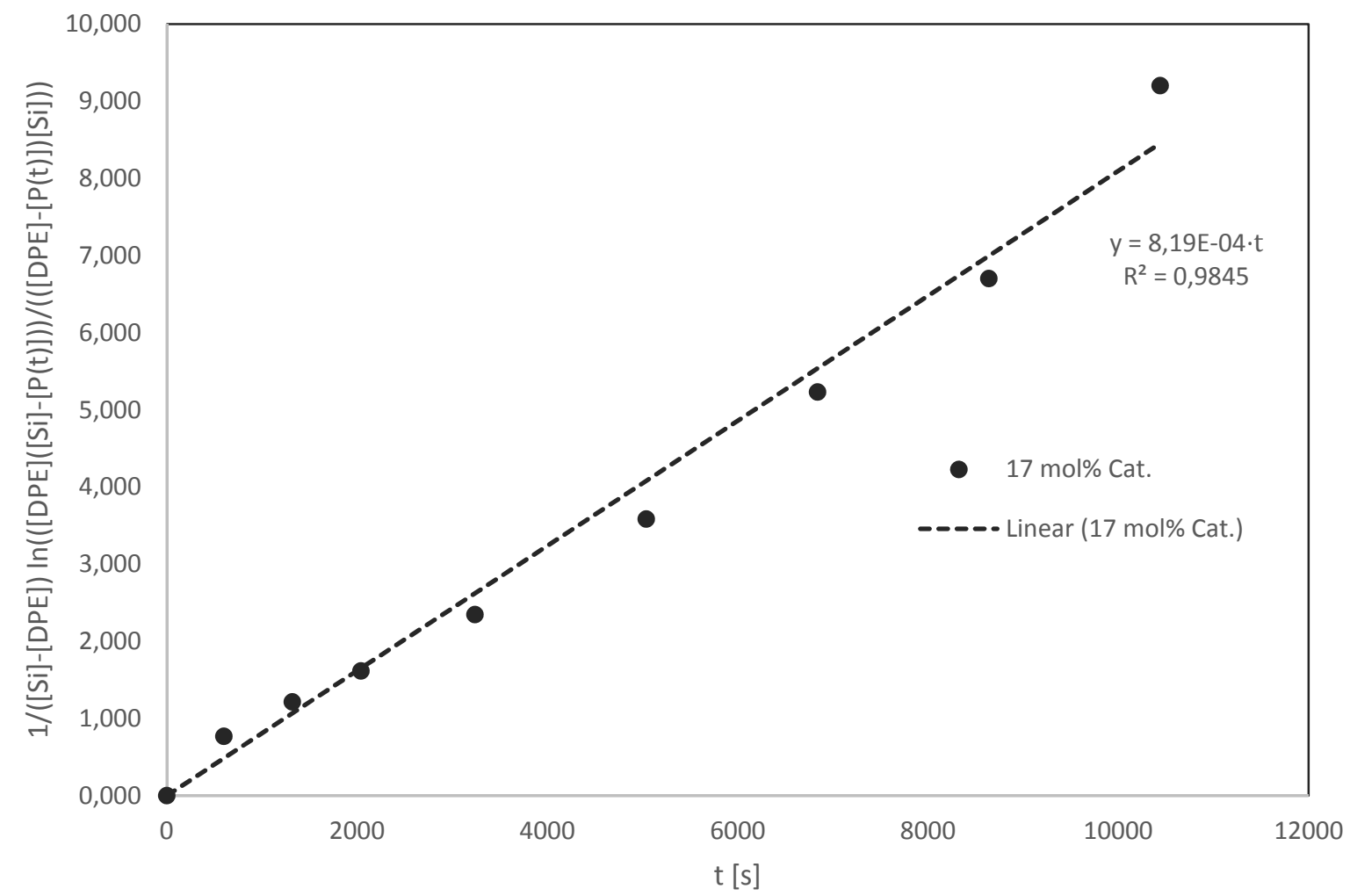

Figure S30. Logarithmic plot of product formation (up to $60 \%$ conversion) with $\mathrm{DSiPh}_{3}$. 


\section{References}

(S1) Leich, V.; Lamberts, K.; Spaniol, T. P.; Okuda, J. Dalton Trans. 2014, 43, 14315-14321. (S2) Smart, K. A.; Mothes-Martin, E.; Annaka, T.; Grellier, M.; Sabo-Etienne, S. Adv. Synth. Catal. 2014, 356, 759-764.

(S3) Wu, T. C.; Wittenberg, D.; Gilman, H. J. Org. Chem. 1960, 25, 596-598.

(S4) Corriu, R. J. P.; Guerin, C.; Henner, B. J. L.; Wang, Q. Organometallics 1991, 10, 35743581.

(S5) Bruker, SAINT+ (Version 7.68); Bruker AXS Inc.: Madison, Wisconsin, USA, 2009.

(S6) Spek, A. L. J. Appl. Crystallogr. 2003, 36, 7-13.

(S7) Altomare, A. Cascarano, G.; Giacovazzo, C.; Guagliardi, A.; Burla, M. C.; Polidori, G.; Camalli, M. J. Appl. Crystallogr. 1994, 27, 435-436.

(S8) Sheldrick, G. M. Acta Crystallogr., Sect. A: Found. Crystallogr. 2008, 64, 112-122.

(S9) Farrugia, L. J. J. Appl. Crystallogr. 2012, 45, 849-854.

(S10) Diamond 4.0.3, Crystal Impact GbR: Bonn, Germany, 2015. 